\title{
Computation of Mixed Type Functional Differential Boundary Value Problems*
}

\author{
Kate A. Abell ${ }^{\dagger}$, Christopher E. Elmer ${ }^{\ddagger}$, A. R. Humphries ${ }^{\S}$, and Erik S. Van Vleck
}

\begin{abstract}
We study boundary value differential-difference equations where the difference terms may contain both advances and delays. Special attention is paid to connecting orbits, in particular to the modeling of the tails after truncation to a finite interval, and we reformulate these problems as functional differential equations over a bounded domain. Connecting orbits are computed for several such problems including discrete Nagumo equations, an Ising model, and Frenkel-Kontorova type equations. We describe the collocation boundary value problem code used to compute these solutions, and the numerical analysis issues which arise, including linear algebra, boundary functions and conditions, and convergence theory for the collocation approximation on finite intervals.
\end{abstract}

Key words. mixed type functional differential equations, boundary value problems, traveling waves, collocation

AMS subject classifications. 65L10, 65L20, 35K57, 74N99

DOI. $10.1137 / 040603425$

1. Introduction. Nonlinear spatially discrete diffusion equations occur as models in many areas of science and engineering. When the underlying mathematical models contain difference terms or delays as well as derivative terms, the resulting differential-difference equations present challenging analytical and computational problems. We demonstrate how functional differential boundary value problems with advances and delays arise from such models and describe a general approach for the numerical computation of solutions. Solutions are approximated for several such problems, and the numerical issues arising in their computation are discussed.

Biology, materials science, and solid state physics are three fields in which accurate first principle mathematical models possess difference (both delayed and advanced) terms. In biology (in particular, in physiology) there is the bidomain model for cardiac tissue (defibrillation), ionic conductance in motor nerves of vertebrates (saltatory conduction), tissue filtration, gas exchange in lungs, and calcium dynamics. Materials science applications include interface

\footnotetext{
${ }^{*}$ Received by the editors January 21, 2004; accepted for publication (in revised form) by W. Beyn March 8, 2005; published electronically September 21, 2004.

http://www.siam.org/journals/siads/4-3/60342.html

${ }^{\dagger}$ Formerly at School of Mathematical Sciences, University of Sussex, Brighton BN1 9QH, UK. Supported by the EPSRC of the UK under Research Studentship \# 97004592.

${ }^{\ddagger}$ Department of Mathematical Sciences, New Jersey Institute of Technology, Newark, NJ 07102 (elmer@m.njit. edu). Supported in part by NSF under grant DMS-0204573.

${ }^{\S}$ Department of Mathematics and Statistics, McGill University, Montreal, Quebec H3A 2K6, Canada (Tony. Humphries@mcgill.ca). Supported by NSERC of Canada, by EPSRC grants GR/M06925 and GR/M87955, NATO under grant CRG971592, and during a visit to the Fields Institute, Toronto, Canada, 2001 by the Leverhulme Trust under a Study Abroad Fellowship and the Fields Institute.

IDepartment of Mathematics, University of Kansas, Lawrence, KS 66045 (evanvleck@math.ku.edu). Supported in part by NATO under grant CRG971592 and by NSF under grants DMS-9973393 and DMS-0139824, and during a visit to the University of Sussex by EPSRC Visiting Fellowship grant GR/M87955.
} 
motion in crystalline materials (crystal growth) and grain boundary movement in thin films where spatially discrete diffusion operators allow description of the material being modeled in terms of its underlying crystalline lattice. In solid state physics, applications include dislocation in a crystal, adsorbate layers on a crystal surface, ionic conductors, glassy materials, charge density wave transport, chains of coupled Josephson junctions, and sliding friction. In all of these fields the physical system, and the corresponding differential model with delay terms, exhibit propagation failure (crystallographic pinning, a mobility threshold) and directional dependence (lattice anisotropy) in a "natural" way. These phenomena do not occur "naturally" in the models without difference terms commonly used for the above applications and are often added to such local models in an ad hoc manner. The reason discrete phenomena are modeled with continuous models is the lack of analytical techniques and numerical solvers for differential equations with both forward and backward delays.

We consider differential-difference boundary value problems. While the applications above are both time (continuous) and space (discrete) dependent, traveling wave solutions are a fundamental class of solutions. Traveling wave solutions for these models satisfy ODEs with advance and delay terms. Thus we consider systems of $d$ mixed type delay equations (see $[34,35,29])$ of the form

$$
\left\{\begin{aligned}
\tau_{I} \mathcal{D}^{\left(m_{I}\right)} u_{I} & =F_{I}(x, \mathbf{u}, \mathbf{y}[\mathbf{u}], \overline{\mathbf{u}}, \overline{\mathbf{y}}[\overline{\mathbf{u}}]), & & I=1, \ldots, d, \quad x \in\left(T_{-}, T_{+}\right), \\
0 & =G_{J}\left(\zeta_{J}, \mathbf{y}[\mathbf{u}]\right), & & J=1, \ldots, m^{*}
\end{aligned}\right.
$$

where for $I=1, \ldots, d, x \in\left(T_{-}, T_{+}\right)$,

$* \tau_{I}(x): \mathbb{R} \rightarrow \mathbb{R}^{+} \cup\{0\}, u_{I}(x): \mathbb{R} \rightarrow \mathbb{R}$,

$* m_{I} \in \mathbb{Z}^{+}$is the order of the $I$ th delay equation,

$* m^{*}$ is the sum of the orders of the delay equations, $m^{*}:=m_{1}+m_{2}+\cdots+m_{d}$,

$* \mathcal{D}^{l} u(x)$ stands for $l$-fold differentiation of $u$ with respect to $x$,

$* \mathbf{u}(x)=\left[u_{1}(x), u_{2}(x), \ldots, u_{d}(x)\right]^{T}$,

* $\mathbf{y}[\mathbf{u}(x)]=\left[u_{1}(x), \mathcal{D} u_{1}(x), \ldots, \mathcal{D}^{\left(m_{1}-1\right)} u_{1}(x), \ldots, u_{d}(x), \mathcal{D} u_{d}(x), \ldots, \mathcal{D}^{\left(m_{d}-1\right)} u_{d}(x)\right]$,

$* \overline{\mathbf{u}}(x)=\left[\overline{\mathbf{u}}_{1}(x), \overline{\mathbf{u}}_{2}(x), \ldots, \overline{\mathbf{u}}_{d}(x)\right]^{T}$,

$* \overline{\mathbf{u}}_{I}(x)=\left[u_{I}\left(x+s_{1}(x)\right), \ldots, u_{I}\left(x+s_{n}(x)\right)\right]^{T}$, where $\left\{s_{j}(x)\right\}_{j=1}^{n}$ is a finite collection of delays, possibly dependent on $x$,

* $\overline{\mathbf{y}}[\overline{\mathbf{u}}(x)]=\left[\bar{u}_{1}(x), \mathcal{D} \bar{u}_{1}(x), \ldots, \mathcal{D}^{\left(m_{1}\right)} \bar{u}_{1}(x), \ldots, \bar{u}_{d}(x), \ldots, \mathcal{D}^{\left(m_{d}\right)} \bar{u}_{d}(x)\right]$, and

$*$ the boundary points satisfy $T_{-} \leqslant \zeta_{1} \leqslant \cdots \leqslant \zeta_{m^{*}} \leqslant T_{+}$.

There is not a well-established general existence and uniqueness theory of traveling waves for the class of problems we are interested in. Initial work was done by Rustichini in [34] and [35]. Mallet-Paret [29] has set forth a linear Fredholm theory which, together with essentially the implicit function theory, establishes an existence theory [30] for a class of differential-delay equations with delays of mixed type (both forward and backward delays).

Previous work finding explicit analytically obtained traveling wave solutions include [12], where traveling wave solutions of a two-dimensional spatially discrete reaction-diffusion system with an idealized, piecewise linear, nonlinear term were studied using Fourier series techniques to determine an integral form of the plane wave solutions. This integral solution was used to relate the wavespeed $c$ to a detuning parameter which allowed the dependence of the behavior on the detuning parameter and the orientation of the wave to be studied. These ideas were 
extended to a general discrete-continuous reaction-diffusion wave equation in [18] and to a problem with variable but spatially periodic diffusion in [19].

In $[18,20]$ numerical techniques were introduced to find traveling wave solutions for

$$
\alpha \dot{u}(\eta, t)+\beta \ddot{u}(\eta, t)=\gamma \Delta u(\eta, t)+L_{D} u(\eta, t)-f(u(\eta, t)),
$$

where $u: \mathbb{R}^{N} \times \mathbb{R} \rightarrow \mathbb{R}, \alpha, \beta, \gamma \geqslant 0, f$ is a bistable nonlinearity, $\Delta$ represents the continuous Laplacian operator, and $L_{D}$ is a discrete Laplacian operator of the form

$$
L_{D} u(\eta, t)=\sum_{k=1}^{N} \varepsilon_{k}\left[u\left(\eta+e_{k}, t\right)+u\left(\eta-e_{k}, t\right)-2 u(\eta, t)\right],
$$

where $\varepsilon_{k} \geqslant 0$ and $e_{k}$ is the unit vector whose $k$ th element equals 1 . Traveling wave solutions of (1.2) of the form

$$
u(\eta, t)=\varphi(\eta \cdot \sigma-c t), \quad \varphi: \mathbb{R} \rightarrow \mathbb{R},
$$

were considered, where $\eta \cdot \sigma$ is the Euclidean dot product of the position vector $\eta$ and the unit vector $\sigma$ normal to the wavefront which indicates the direction of the traveling wave with respect to the lattice, and $c \in \mathbb{R}$ is the unknown wavespeed.

Substituting the traveling wave ansatz (1.3) into (1.2) results in

$$
-c \alpha \varphi^{\prime}(\xi)-\left(\gamma-c^{2} \beta\right) \varphi^{\prime \prime}(\xi)=L_{T} \varphi(\xi)-f(\varphi(\xi)), \quad \xi \in \mathbb{R}
$$

where $\xi=\eta \cdot \sigma-c t \in \mathbb{R}$, and

$$
L_{T} \varphi(\xi)=\sum_{k=1}^{N} \varepsilon_{k}\left[\varphi\left(\xi+e_{k} \cdot \sigma\right)-2 \varphi(\xi)+\varphi\left(\xi-e_{k} \cdot \sigma\right)\right] .
$$

Note that (1.4) is a delay differential equation (DDE) of mixed type posed on an infinite interval, with both delayed and advanced terms contained in (1.5) as a result of applying the traveling wave ansatz to the discrete Laplacian operator.

In [18] equations (1.4) and (1.5) are solved on a truncated interval $\xi \in[-T, T]$, using asymptotic boundary conditions (see [28]). An ordinary differential boundary value problem solver was used, together with path following techniques to find solutions in different regions of the parameter space. The main difficulty with this approach is the delayed and advanced terms in (1.5). The simple approach, of treating these terms as source terms in a fixed point iteration was adopted in [18], resulting in an iterative scheme. The methods used in [18] become increasingly inefficient as $\left(\gamma-c^{2} \beta\right) \rightarrow 0$ and are nonconvergent for $\left(\gamma-c^{2} \beta\right)=0$ in (1.4). Thus they could not be used for computing traveling wave solutions of the Nagumo equation obtained by setting $\beta=\gamma=0$ in (1.4). In [20] the introduction of a Newton-like iterative scheme allows general bistable nonlinearities $f$ to be considered. The convergence results in [20], based upon the Fredholm theory of Mallet-Paret [29, 30], provide a theoretical foundation for the Newton-like method considered in this paper.

The approximation of periodic solutions and connecting orbits of DDEs has been considered in $[22,21,36]$, where the problems are formulated as boundary value problems and 
approximated with collocation methods. In addition, the stability of a class of collocation methods is analyzed in [21], convergence is shown to correspond to known convergence results for initial value delay equations in [22], and a technique for approximating connecting orbits for DDEs with stable or unstable manifolds of infinite dimension is developed in [36].

The outline of this paper is as follows. In section 2 we describe how traveling wave problems in lattice differential equations result in functional differential equations of the form (1.1). Although there is now extensive theory for traveling wave solutions of parabolic type PDEs, relatively little is known about traveling wave solutions of spatially discrete analogues, because of the difficulty of solving (1.1). In section 3 we will present numerically computed solutions to problems of the form (1.1) arising from several traveling wave problems. These include in section 3.1 a spatially discrete Nagumo equation with piecewise linear nonlinearity, and in section 3.2 the spatially discrete Nagumo problem ((1.2) with $\beta=\gamma=0, \alpha=1$, and cubic nonlinearity). In section 3.3 we present a discrete Nagumo equation with a cubic-like nonlinearity for which we have exact traveling wave solutions, and we use this to illustrate and numerically verify the performance of our code. The numerical results show proportionality of the error with the requested tolerance. In sections 3.4 and 3.5 an Ising model, where $F_{I}$ is a fully nonlinear function of $u$ and its delays, and Frenkel-Kontorova (FK) type equations with periodic boundary conditions are considered. In section 4 we describe the main features and implementation of our general-purpose code, COLMTFDE (COLlocation for Mixed Type Functional Differential Equations), a collocation-based boundary value problem solver for the solution of linear and nonlinear differential-difference equations with both advances and delays that was used to perform the computations in section 3. This code is a member of the COLSYS family $[2,6,13,42]$ but is differentiated from other members of the family by its ability to handle delays and several other features that we describe. Details of the collocation formulation are given in section 4.1, and convergence theory for the collocation error on finite intervals, due to Bader, is summarized in section 4.2. We do not provide convergence results, theoretically or numerically, for the full discretization, the approximations due to collocation and the truncation to a finite interval.

\section{Traveling wave solutions.}

2.1. Connecting orbits. We will numerically solve parameterized boundary value problems of the forms

$$
\dot{\varphi}(\xi)=\bar{g}\left(\xi, \varphi(\xi), \varphi\left(\xi+s_{1}\right), \ldots, \varphi\left(\xi+s_{n}\right), \lambda\right), \quad \lim _{\xi \rightarrow-\infty} \varphi(\xi)=\varphi_{l}, \quad \lim _{\xi \rightarrow \infty} \varphi(\xi)=\varphi_{r}
$$

and

$$
\ddot{\varphi}(\xi)=\bar{g}\left(\xi, \varphi(\xi), \dot{\varphi}(\xi), \varphi\left(\xi+s_{1}\right), \ldots, \varphi\left(\xi+s_{n}\right), \lambda\right), \quad \lim _{\xi \rightarrow-\infty} \varphi(\xi)=\varphi_{l}, \quad \lim _{\xi \rightarrow \infty} \varphi(\xi)=\varphi_{r},
$$

where $\varphi(\xi): \mathbb{R} \rightarrow \mathbb{R}$ and $\lambda \in \mathbb{R}^{p}$, whose solution $\left(\varphi_{0}, \lambda_{0}\right)$ is an orbit connecting $\varphi_{l}$ and $\varphi_{r}$.

Recall that applying the traveling wave ansatz (1.3) to (1.2) results in (1.4), where $L_{T}$ is defined by (1.5), although we often take the simpler form

$$
L_{T} \varphi(\xi)=L_{1} \varphi(\xi)=(\varphi(\xi+1)-2 \varphi(\xi)+\varphi(\xi-1)) .
$$


With the unknown wavespeed $c$ taking the role of $\lambda,(1.4)$ is of the form (2.1) or (2.2), where to satisfy the boundary conditions we require $f\left(\varphi_{l}\right)=f\left(\varphi_{r}\right)=0$. We will see such $f$ in section 3 .

2.2. Fredholm theory, existence, and stability. Consider the variation of the connecting orbit problem (2.1) with respect to $\varphi$ and $\lambda$,

$$
\dot{\psi}(\xi)=\bar{g}_{\varphi}\left(\xi, \bar{\varphi}_{0}(\xi), \lambda_{0}\right) \psi(\xi)+\bar{g}_{\lambda}\left(\xi, \bar{\varphi}_{0}(\xi), \lambda_{0}\right) \mu, \quad \psi( \pm \infty)=0,
$$

where $\bar{\varphi}_{0}(\xi)=\left(\varphi_{0}(\xi), \varphi_{0}\left(\xi+s_{1}\right), \ldots, \varphi_{0}\left(\xi+s_{n}\right)\right)$ and observe that $\left(\psi=b \dot{\varphi}_{0}, \mu=\dot{\lambda}_{0}=0\right)$ is an isolated solution for some $b \in \mathbb{R}$. Note that (2.4) is a condition for a nondegenerate connecting orbit between fixed points [11], i.e., a well-defined traveling wave problem. Numerically we deal with this translational invariance by imposing a phase condition, $\Phi$, which maps the Banach space of continuous solutions to (2.1) into $\mathbb{R}$ and satisfies

$$
\Phi\left(\varphi_{0}, \lambda_{0}\right)=0, \quad \Phi_{\varphi}\left(\varphi_{0}, \lambda_{0}\right) \dot{\varphi}_{0}+\Phi_{\lambda}\left(\varphi_{0}, \lambda_{0}\right) \dot{\lambda}_{0}=\Phi_{\varphi}\left(\varphi_{0}, \lambda_{0}\right) \dot{\varphi}_{0} \neq 0 .
$$

The "well posedness" of the mixed type delay equation (2.1) with (2.5) has been established by Mallet-Paret $[29,30]$ in analogy with the Fredholm theory of Palmer [33] (see also Beyn [11]) for differential equations. This requires consideration of asymptotic hyperbolicity, establishing the correct relationship between the number parameters in the system and the dimension of stable and unstable subspaces of the asymptotic operators to obtain a Fredholm index of zero and hence an isomorphic map, and essentially involves establishing an exponential dichotomy.

In [29] Mallet-Paret develops a Fredholm theory for mixed type delay equations like those considered here. Subsequently, in [30] he employs this Fredholm theory to prove existence, uniqueness (up to translation), and other properties of monotone traveling wave solutions of spatially discrete Nagumo equations. We now summarize important points of this Fredholm theory. Consider the linear operator $\Lambda_{L}: W^{1, p} \rightarrow L^{p}$ given by

$$
\left(\Lambda_{L} x\right)(\xi)=x^{\prime}(\xi)-\sum_{j=0}^{n} A_{j}(\xi) x\left(\xi+s_{j}\right), \quad \xi \in J,
$$

where $J$ is typically the infinite interval, and the $A_{j}$ are $d \times d$ measurable, locally integrable, complex matrices. The shifts $s_{j}$ may be positive or negative and it is assumed that $s_{0}=0$ and that the shifts are distinct.

Theorem A of [29] is a Fredholm alternative theorem for linear mixed type delay equations that states that $\Lambda_{L}$ is Fredholm if $\Lambda_{L}$ is asymptotically hyperbolic; i.e., the limiting operators (as $\xi \rightarrow \pm \infty$ ) are constant and the corresponding characteristic equations have no solutions of the form $i \eta$ for $\eta \in \mathbb{R}$. Because the dimensions of the unstable manifolds of the limiting operators may be infinite, the Fredholm index is calculated (see Theorem C of [29]) using a homotopy between the limiting operators.

We mention here that a stability theory is developed in [14] in the spirit of that of Fife and McLeod [24] for the Nagumo PDE that shows that traveling wave solutions of the spatially discrete Nagumo equation are asymptotically stable with asymptotic phase. Exponential dichotomies have been established for linear nonautonomous mixed type functional differential equations [25, 31] and together with the Fredholm theory of Mallet-Paret $[29,30]$ results in the spirit of Sandstede [37] (see also [38]) appear possible. 
2.3. Boundary functions and boundary conditions. To find traveling wave solutions of lattice differential equations numerically, we truncate the infinite interval $(-\infty, \infty)$ to a finite interval $\left(T_{-}, T_{+}\right)$and solve the differential equation numerically on this interval.

If the functional differential equation (1.1) has delays which satisfy $s \in\left[s_{\min }, s_{\max }\right]$ where $s_{\max } \leqslant 0$ so there are no advanced terms, then boundary functions $u_{I}(x)$ can be defined on the interval $\left[T_{-}+s_{\min }, T_{-}\right]$, so that $F_{I}$ can be evaluated at all points $x \in\left(T_{-}, T_{+}\right)$. When such functions are defined delay differential equations may be solved as initial value problems. However, the connecting orbit problem with delays only can also be considered as a boundary value problem [36]. In this case either the stable or the unstable manifold is finite dimensional, and, as is shown in [36], given enough free parameters one of two choices will result in a finite number of conditions: (i) expansion in terms of eigenfunctions, or (ii) the use of a special bilinear form to define a complementary projection.

However we consider problems with $0 \in\left(s_{\min }, s_{\max }\right)$ and so require boundary functions to be defined on both of the intervals $\left[T_{-}+s_{\min }, T_{-}\right]$and $\left[T_{+}, T_{+}+s_{\max }\right]$ in order to be able to compute the collocation solution. In this case the stable and unstable manifolds of the equilibrium solutions are generally both infinite dimensional. Moreover, the solutions on the interval $\left[T_{-}, T_{+}\right]$and on the boundary intervals are interdependent, so not only do we require boundary functions consistent with the boundary conditions at $\pm \infty$, but we must find these functions while simultaneously solving on $\left[T_{-}, T_{+}\right]$.

We work with implicitly defined boundary functions which we define using ideas similar to those for asymptotic boundary conditions for differential equations on infinite intervals (see $[15,28])$. For $x<T_{-}$we define the boundary function

$$
u_{I}(x)=H_{-}\left(u_{I}\left(x_{s}\right), \mathcal{D} u_{I}\left(x_{s}\right), \ldots, \mathcal{D}^{(m-1)} u_{I}\left(x_{s}\right)\right), \quad x<T_{-}, \quad x_{s} \in\left[T_{-}, T_{+}\right] .
$$

Here $x_{s}$ is usually taken to be $T_{-}$. Thus the value of the boundary function $u_{I}(x)$ is defined in terms of the unknowns $u_{I}\left(T_{-}\right)$and its derivatives. Similarly we define a separate boundary function $H_{+}(x)$ for $x>T_{+}$.

We usually find such a representation for the solution outside the interval using a technique similar to eigenvector boundary conditions. For example, consider the differential-difference equation

$$
-c \dot{\varphi}(\xi)=L_{1} \varphi(\xi)-f(\varphi(\xi))
$$

with $L_{1}$ given by (2.3). Truncating to a finite interval, the form but not the magnitude of the solution outside the computational domain can be found by linearization. Imposing continuity conditions between the numerically computed and linearized parts of the solution then allows us to simultaneously solve the boundary value problem and determine the magnitude of the boundary function to ensure that desired continuity properties hold at the boundary.

Consider the linearization of (2.8) about the equilibrium points $\varphi_{l}$ and $\varphi_{r}$,

$$
-c \dot{v}(\xi)=\alpha L_{1} v(\xi)-\beta v(\xi)
$$

where $\beta=f^{\prime}\left(\varphi_{l}\right)$ or $\beta=f^{\prime}\left(\varphi_{r}\right)$. The characteristic equation for (2.9) is obtained by substituting $v(\xi)=\exp (\lambda \xi)$ into $(2.9)$,

$$
h(\lambda)=c \lambda+\alpha(\exp (\lambda)-2+\exp (-\lambda))-\beta=2 \alpha \cosh (\lambda)+c \lambda-(2 \alpha+\beta)=0,
$$


which for $\alpha>0$ and $\beta>0$ has two real solutions, one positive and one negative. Denote by $\lambda_{0}^{+}$and $\lambda_{0}^{-}$the positive and negative real roots, respectively, of $(2.10)$ for $\beta=f^{\prime}\left(\varphi_{l}\right)$ and by $\lambda_{1}^{+}$and $\lambda_{1}^{-}$the positive and negative roots for $\beta=f^{\prime}\left(\varphi_{r}\right)$.

Now consider truncating (2.8) with $\dot{c}=0$ and a phase condition to a finite time interval $\left[T_{-}, T_{+}\right]$and then consider the following truncated problem:

$$
\left\{\begin{array}{l}
-c \dot{\varphi}_{\pi}(\xi)=\alpha L_{1} \varphi_{\pi}(\xi)-f\left(\varphi_{\pi}(\xi)\right), \quad T_{-}<\xi<T_{+}, \\
\varphi_{\pi}(\xi)=1+\left(\varphi_{\pi}\left(T_{+}\right)-1\right) \mathrm{e}^{\lambda_{1}^{-}\left(\xi-T_{+}\right)}, \quad \xi \in\left[T_{+}, \infty\right), \\
\varphi_{\pi}(\xi)=\varphi_{\pi}\left(T_{-}\right) \mathrm{e}^{\lambda_{0}^{+}\left(\xi-T_{-}\right)}, \quad \xi \in\left(-\infty, T_{-}\right], \\
-\dot{\varphi}_{\pi}\left(T_{+}\right)+\lambda_{1}^{-} \varphi_{\pi}\left(T_{+}\right)=\lambda_{1}^{-}, \\
-\dot{\varphi}_{\pi}\left(T_{-}\right)+\lambda_{0}^{+} \varphi_{\pi}\left(T_{-}\right)=0 .
\end{array}\right.
$$

The second and third equations in (2.11) give us a representation for $\varphi_{\pi}$ outside of $\left[T_{-}, T_{+}\right]$in terms of $\varphi_{\pi}$ at the boundary and the eigenvalues and are determined by imposing continuity in $\varphi_{\pi}$ at the boundary. Also, the fourth and fifth equations, which are the boundary conditions, come from requiring continuity in the derivative, since differentiating the second equation gives

$$
\varphi_{\pi}^{\prime}(\xi)=\lambda_{1}^{-}\left(\varphi_{\pi}\left(T_{+}\right)-1\right) \mathrm{e}^{\lambda_{1}^{-}\left(\xi-T_{+}\right)},
$$

and hence

$$
\varphi_{\pi}^{\prime}\left(T_{+}\right)=\lambda_{1}^{-}\left(\varphi_{\pi}\left(T_{+}\right)-1\right)
$$

which gives the fourth equation in (2.11). The fifth equation is derived similarly.

The left and right boundary functions defined in (2.11) for $\xi \leqslant T_{-}$and $\xi \geqslant T_{+}$are thus each composed of a single monotonic eigenfunction, whereas (2.10) is transcendental and has infinitely many complex conjugate solutions. However in each of the Nagumo and Ising problems considered in sections 3.1-3.4 the real roots of (2.10) and its analogues are not only dominant, but simple, and there is a gap in the real part compared with any other solution to the characteristic equation. Thus any expansion in terms of eigenfunctions has a leading order term corresponding to the appropriate real solution of the characteristic equation. For problems where the dominant roots of the characteristic equation are complex conjugate or where there is no spectral gap, a different form of boundary function would be required.

In our computations the values $\lambda_{0}^{+}$and $\lambda_{1}^{-}$are found numerically. In order to bracket a root of $h(\lambda)$ in (2.10) notice that $h(0)<0$ since $\beta>0$. Consider $(2.10)$ with $\cosh (\lambda)$ replaced with $\lambda^{2}$ and call this quadratic that has one negative and one positive root $\hat{h}(\lambda)$. Then for $\hat{h}\left(\lambda^{*}\right)=0$ we have $($ since $\alpha>0)$ that $h\left(\lambda^{*}\right)>0$. Thus, we bracket a solution of $h(\lambda)=0$ and employ the code zero of [39] that uses a combined bisection/secant method strategy to numerically solve the nonlinear equation for the desired $\lambda$. We also note here that when linearizing with respect to the wavespeed $c$ we employ the identity $\frac{\partial \lambda}{\partial c}=-\frac{\partial h}{\partial c} / \frac{\partial h}{\partial \lambda}$.

2.4. Phase condition and wavespeed. DDE boundary value problems defined on an infinite interval, or with periodic boundary conditions, exhibit translational invariance. To 
determine a unique translate of the wave form a phase condition is required. Let $\varphi_{0}$ be a reference wave form; we consider the classical phase condition

$$
\varphi(0)=\varphi_{0}(0),
$$

which assumes that both $\dot{\varphi}(0)$ and $\dot{\varphi}_{0}(0)$ are nonzero. In our computations we need to solve for both the waveform $\varphi$ and the wavespeed $c$. We do this by approximating (2.11) together with the classical phase condition (2.12) and the equation $\dot{c}(\xi)=0$.

3. Solutions. We demonstrate numerically computed solutions to a number of instances of (1.1). We begin in section 3.1 with a piecewise linear control problem for which exact solutions are known. In section 3.2 we compute solutions of a nonlinear discrete Nagumo equation, which had previously only been solved under the addition of artificial diffusion. In section 3.3 we present a cubic-like nonlinearity for which we have exact traveling solutions to a discrete Nagumo equation. We use this example to illustrate and numerically verify the performance of our code. In section 3.4 we compute solutions of an Ising model where the $F_{I}$ is a fully nonlinear function of $u$ at its delays. In section 3.5 we solve an FK type equation with periodic boundary conditions.

3.1. Piecewise linear spatially discrete reaction-diffusion equation. We consider the traveling wave equations that result from applying the traveling wave ansatz (1.3) to the spatially discrete evolution equation (1.2) with $\alpha=1, \beta=0$, and with $f$ given by the piecewise linear nonlinearity ("McKean's caricature of the cubic" [32])

$$
f(\varphi) \equiv f(\varphi, a)=\left\{\begin{array}{cl}
\varphi, & \varphi<a, \\
{[\varphi-1, \varphi],} & \varphi=a, \\
\varphi-1, & \varphi>a,
\end{array} \quad a \in(0,1) .\right.
$$

The resulting equations for this spatially discrete Nagumo equation are

$$
\left\{\begin{array}{c}
-c \dot{\varphi}(\xi)=\alpha L_{1} \varphi(\xi)+\gamma \ddot{\varphi}(\xi)-f(\varphi(\xi)), \\
\varphi(-\infty)=0, \varphi(0)=a, \varphi(+\infty)=1
\end{array}\right.
$$

where $L_{1} \varphi$ is defined by (2.3). If $\varphi$ is monotone, then we may set $\varphi(0)=a$, which implies $\varphi(\xi)<a$ for $\xi<0$ and $\varphi(\xi)>a$ for $\xi>0$. Thus, for the Heaviside function defined by

$$
h(x)= \begin{cases}1, & x>0 \\ 0, & x<0\end{cases}
$$

we have $h(\varphi(\xi)-a)=h(\xi)$ for $\xi \neq 0$, and so $f(\varphi(\xi))=\varphi(\xi)-h(\xi)$. This incorporates the phase condition into our problem, and hence we may solve (3.2) with (3.1) as a linear inhomogeneous equation where $c$ is a given parameter and the corresponding value of $a$ is determined by $\varphi(0)$. We are particularly interested in the case where $\gamma=0$, so that we are solving the purely spatially discrete equation, with no artificial diffusion terms. Hence, (3.2) is reduced to

$$
\left\{\begin{array}{c}
-c \dot{\varphi}(\xi)=\alpha L_{1} \varphi(\xi)-\varphi(\xi)+h(\xi) \\
\varphi(-\infty)=0, \varphi(0)=a, \varphi(+\infty)=1
\end{array}\right.
$$


Since the nonlinearity $f$ is piecewise linear, the exact traveling wave solution to (3.3) can be derived using Fourier transforms (see [12] and [18]). We use our code to compute numerical solutions to this problem. Figure 1(i) shows a plot of $a(c)$ against $c$, obtained numerically for the spatially discrete problem (3.3).
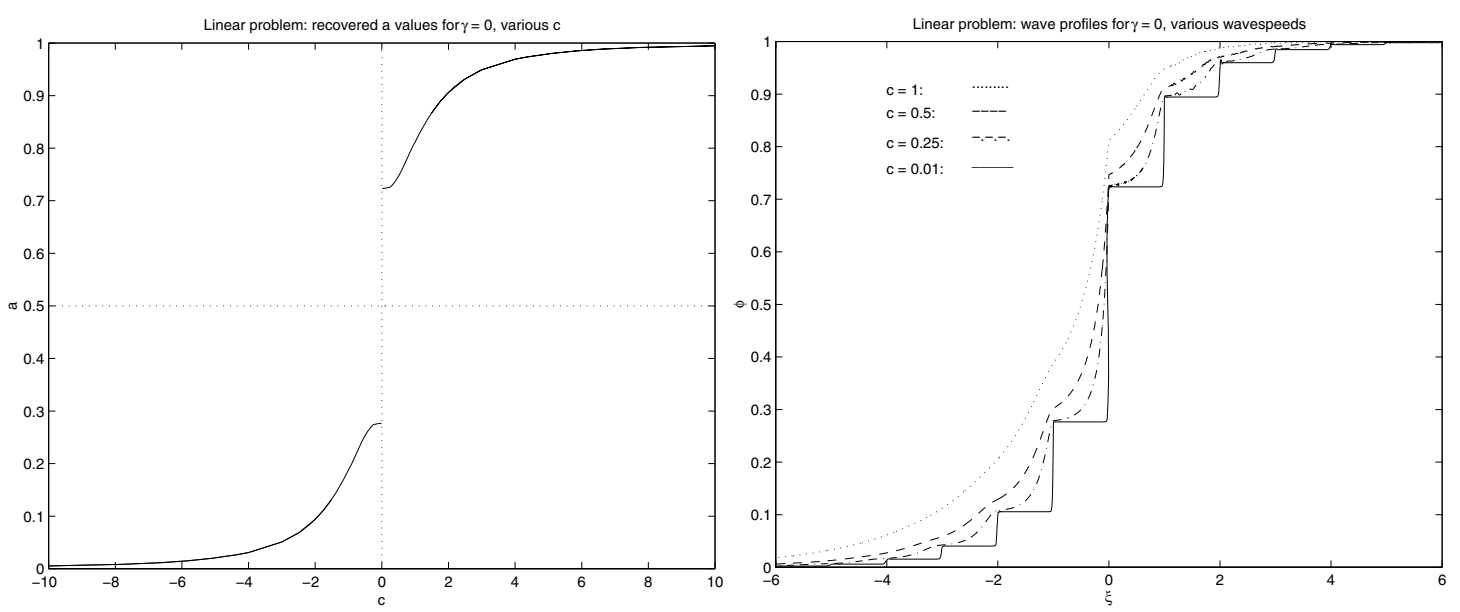

Figure 1. (i) An a(c) plot for the spatially discrete linear reaction-diffusion equation (3.3), where the solution is computed numerically for a specified $c$, and $a$ is recovered using $\varphi(0)=a$. (ii) Wave profiles for the spatially discrete linear reaction-diffusion equation (3.3) for various wavespeeds c.

Figure 1(ii) shows numerically obtained solution profiles of the spatially discrete problem (3.3) for various wavespeeds $c$. We present solution curves only for $a \geqslant 0.5$ because of the symmetry which these solutions possess with the solutions for $a \leqslant 0.5$. Note the "kink" which forms in the solution curves at $\varphi(0)$. The existence of this is discussed in [18] and is due to the fact that taking $\gamma=0$ in (3.2) implies for $c \neq 0$ that

$$
\lim _{\xi \rightarrow 0+} \varphi^{\prime}(\xi) \neq \lim _{\xi \rightarrow 0-} \varphi^{\prime}(\xi) .
$$

Note also from Figure 1(ii) that $c$ is a monotonic increasing function of $\varphi(0)=a$.

The graphs in Figure 1 agree well with the equivalent graphs presented in [18] for the exact form of the solution [18, Figure 2.6, Curve 1] and using the iterative numerical method outlined in the introduction [18, Figures 3.2 and 3.3, Curve 1].

3.2. Spatially discrete Nagumo equation. We now consider the traveling wave equations corresponding to a spatially discrete Nagumo equation

$$
\left\{\begin{array}{c}
-c \dot{\varphi}(\xi)=L_{1} \varphi(\xi)-f(\varphi(\xi)), \\
\varphi(-\infty)=0, \varphi(+\infty)=1
\end{array}\right.
$$

where $L_{1} \varphi$ is defined by (2.3) and the cubic nonlinearity $f$ is defined by

$$
f(\varphi) \equiv f(\varphi, a)=d_{1} \varphi(\varphi-1)(\varphi-a), \quad a \in(0,1)
$$


This problem has been studied by Bell and Cosner [9], Keener [26, 27], Zinner [43, 44], and others. We also mention here the general existence and uniqueness theory based upon a Fredholm alternative theorem of Mallet-Paret [29, 30] and the stability theory of Chow, MalletParet, and Shen [14].

We solve (3.4) with the classical phase condition $\varphi(0)=a$. Since the wavespeed $c$ is unknown, we solve (3.4) simultaneously with the equation $\dot{c}=0$, to obtain both solutions $\varphi$ and $c$. We are therefore actually solving

$$
\left\{\begin{array}{c}
-c \dot{\varphi}(\xi)=L_{1} \varphi(\xi)-d_{1} \varphi(\xi)(\varphi(\xi)-1)(\varphi(\xi)-a), \\
\dot{c}=0, \\
\varphi(-\infty)=0, \varphi(0)=a, \varphi(+\infty)=1 .
\end{array}\right.
$$

Figure 2 shows plots of the detuning parameter $a(c)$ against the wavespeed $c$ for (3.6) with various cubic coefficients $d_{1}$. Propagation failure is the term we use when there is a nontrivial interval (one of nonzero length) of parameter $a$ values for which $c=0$. Propagation failure is resolved numerically if $d_{1}$ is large enough (see [23] for details on the subtleties of the existence of the interval of propagation failure), and the length of this interval increases as $d_{1}$ increases.

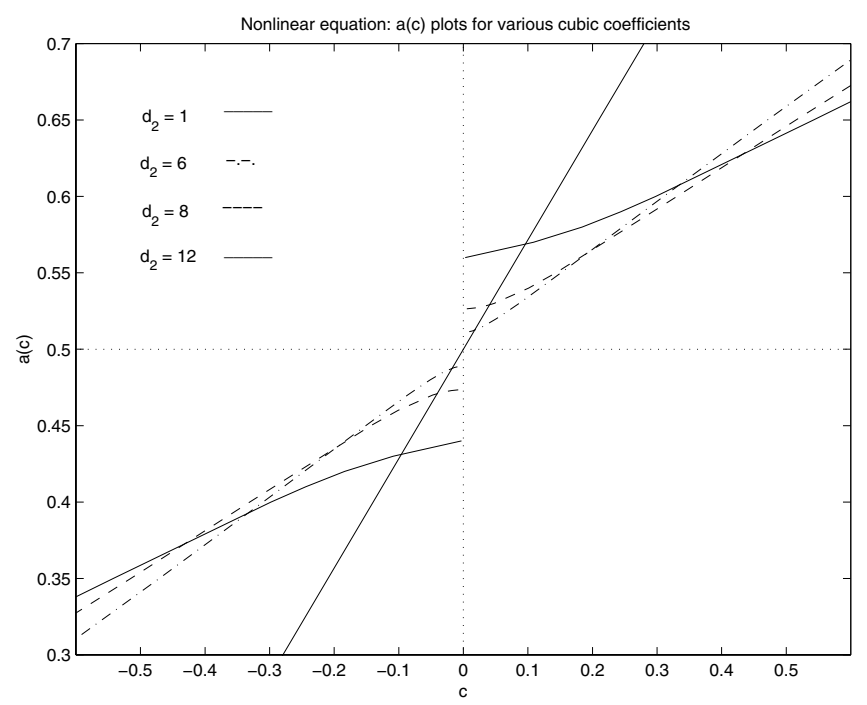

Figure 2. Plots of $a(c)$ against the wavespeed $c$ for the spatially discrete nonlinear reaction-diffusion equation (3.4), showing the dependence of the size of the interval of propagation failure on the cubic coefficient $d_{1}$ using difference operator $L_{1}$ for which $\alpha=1$.

In [20], numerical computations were carried out with an artificial diffusion term $\gamma \ddot{\varphi}$ imposed on the problem to allow it to be solved using an iterative approach. It was shown that for small $\gamma$, for example, $\gamma=10^{-4}$, there existed an interval of $a$ for which $|c|<10^{-3}$, and it was suggested that propagation failure would also be seen for the purely spatially discrete problem, i.e., when $\gamma=0$. We confirm this suggestion in Figure 2.

Figure 3 illustrates the solutions for $a$ outside the interval of propagation failure when $d_{1}=10$. In this case we are able to compute solutions for $|a-0.5| \geqslant 0.045$. As for the linear 

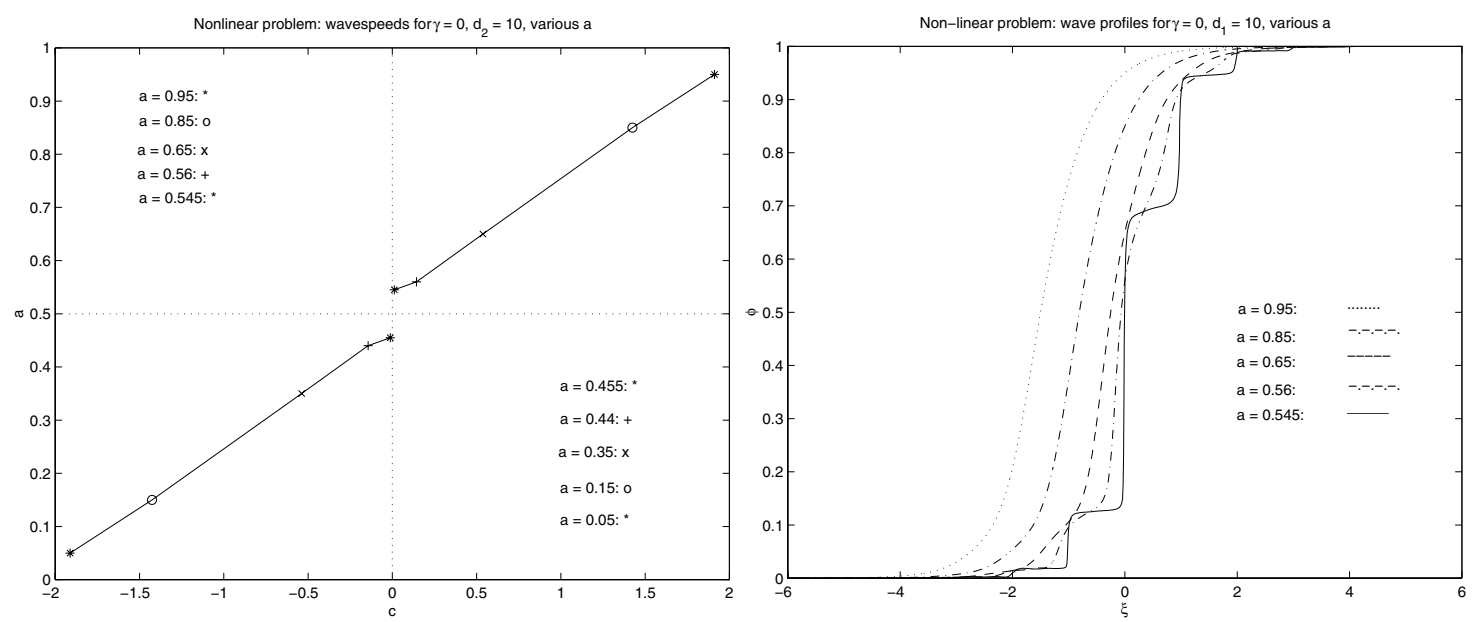

Figure 3. (i) a(c) against c for the spatially discrete Nagumo equation (3.6) and cubic coefficient $d_{1}=10$ for various values of $a$. (ii) The corresponding wave profiles $\varphi(\xi)$.

problem, as $|a-0.5|$ increases toward 0.5 the magnitude of the wavespeed $|c|$ becomes large and the solution has a hyperbolic tangent shape. For values of $|a-0.5|$ close to 0.045 , or in other words, close to the interval of propagation failure, the solutions exhibit step-like behavior and $|c|$ is small. Away from the wave front, the tails of these solutions decay exponentially.

Solving (3.4) for values of the detuning parameter $a$ which lie inside the interval of propagation failure (i.e., $c=0$ ) is a very difficult problem. We follow the approach of [20], and introduce an artificial diffusion term, $\gamma \ddot{\varphi}$, giving

$$
\left\{\begin{array}{c}
0=L_{1} \varphi(\xi)+\gamma \ddot{\varphi}(\xi)-f(\varphi(\xi)), \\
\dot{c}=0, \\
\varphi(-\infty)=0, \varphi(0)=a, \varphi(+\infty)=1 .
\end{array}\right.
$$

In [20] this was solved numerically using an iterative scheme based on COLMOD with the delay terms treated as source terms, for values of $\gamma$ of the order $\gamma=10^{-4}$, but these schemes failed to converge for smaller values of $\gamma$. We are now able to solve (3.7) with $\gamma$ of the order $\gamma=10^{-6}$. An example is given in Figure 4 (observe the $a=0.5$ and the $a=0.54$ curves), where $d_{1}=10$ and various values of the detuning parameter $a$ are considered. While the actual solutions $\varphi$ for these values of $a(0.5$ and 0.54$)$ are discrete maps, our approximate solutions obtained are continuous.

3.3. Spatially discrete Nagumo equation with exact solution. We show by example the numerical accuracy of COLMTFDE with respect to the collocation, and with respect to the truncation of the infinite interval. We proceed by assuming a solution form for the discrete Nagumo equation of section 3.2 and constructing the bistable nonlinearity which gives the chosen solution. Using this example, with various choices of interval length and various numbers of collocation points per mesh interval, we compute numerical solutions and compare the results with the exact solution. 

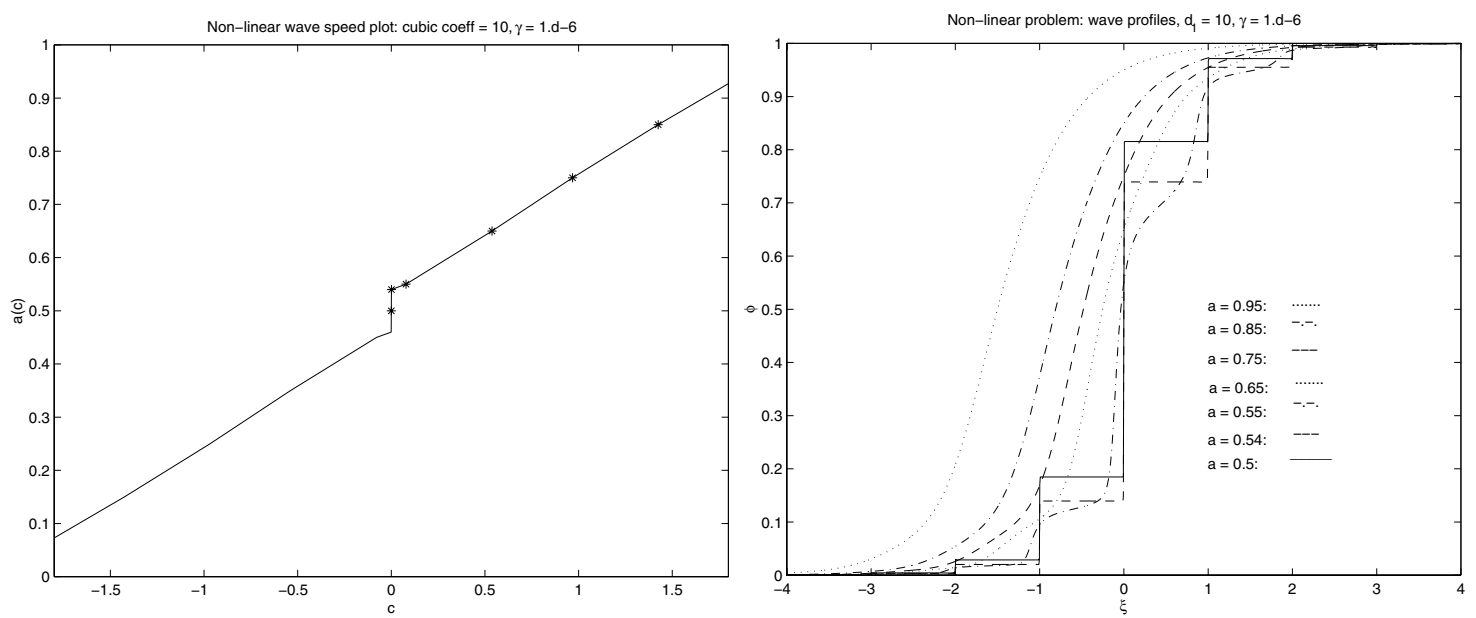

Figure 4. Spatially discrete reaction-diffusion equation (3.7) with cubic nonlinearity (3.5), where $\gamma=10^{-6}$ and cubic coefficient $d_{1}=10$, showing the dependence of the wavespeed and the solution profile on the detuning parameter $a$.

3.3.1. Constructing the equation. We assume that the solution to

$$
\left\{\begin{array}{c}
-c \dot{\varphi}(\xi)=\alpha L_{1} \varphi(\xi)-f(\varphi(\xi)), \quad \text { is of the form } \quad \varphi(\xi)=\frac{1}{2}\left[1+\tanh \frac{1}{2}(b \xi+g(\xi))\right] \\
\varphi(-\infty)=0, \varphi(+\infty)=1,
\end{array}\right.
$$

(see [17]), where $b \xi+g(\xi)$ is a monotone increasing function. This requires that the nonlinearity be

$$
f(\varphi(\xi))=\alpha L_{1} \varphi(\xi)+c\left[b+g^{\prime}(\xi)\right] \varphi(\xi)(1-\varphi(\xi)) \quad \text { with } \quad c=\alpha \frac{\left[2 a-\varphi\left(\xi_{0}+1\right)-\varphi\left(\xi_{0}-1\right)\right]}{\left[b+g^{\prime}\left(\xi_{0}\right)\right] a(1-a)}
$$

where $\xi_{0}$ is the value of $\xi$ such that $\varphi\left(\xi_{0}\right)=a$, enforcing the condition that $0 \equiv f(a)$. The only independent parameters are $\alpha$ and $a$.

In this example we let $b=[1+3(2 a-1)] / \sqrt{2 \alpha}$ and

$$
g(\xi)=\mu\left[\frac{\left(\xi-q_{1}\right)\left(q_{3}-\xi\right)\left(q_{2}-\xi\right)}{\left(\xi-q_{1}\right)\left(q_{3}-\xi\right)+d_{1}}+w\right] .
$$

The parameters $d_{1}, w$, and $\mu$ allow us to adjust the effect and strength of $g$ so that we may produce propagation failure and other effects which are typically present in mixed type functional differential equations. We have chosen

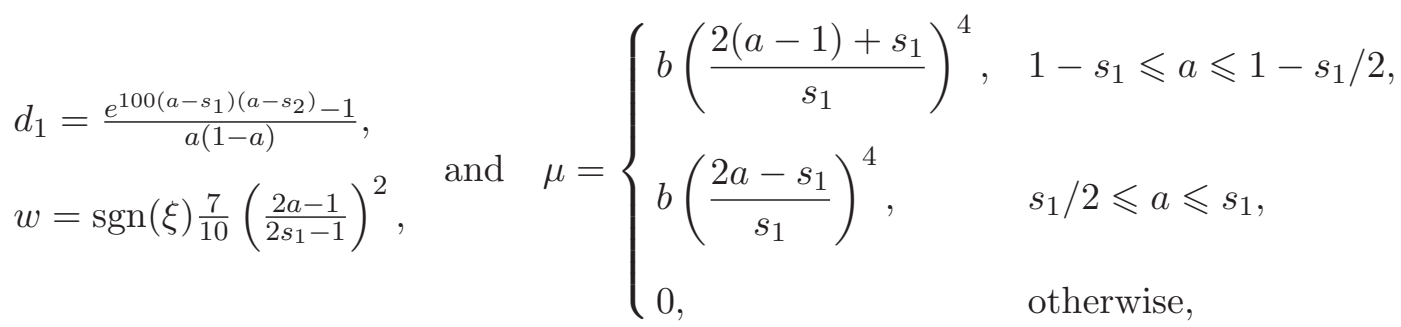


where $s_{1}=\frac{1}{2} e^{-1 /(4 \sqrt{2 \alpha})}, s_{2}=1-s_{1}$. The roots $q_{1}, q_{2}$, and $q_{3}$ are

$$
\begin{aligned}
& q_{1}=n+p, \\
& q_{2}=n+1 / 2+p, \quad n \leqslant \xi<n+1, \quad n \in Z, \quad \text { and } \quad p= \begin{cases}p_{1}, & \xi \leqslant 0 \\
-p_{1}, & \xi>0\end{cases} \\
& q_{3}=n+1+p,
\end{aligned}
$$

where $p_{1}$ is the left most real root of $y^{3}-(3 / 2+w) y^{2}+(1 / 2+w) y+w d_{1}$ and is chosen so that $g(\xi)$ is $C^{1}$.

Suppose $a \notin\left[s_{1} / 2,1-s_{1} / 2\right]$; then $g(\xi)=g^{\prime}(\xi)=0$,

$$
f(\varphi)=\left(\frac{\alpha \gamma(2 \varphi-1)}{\gamma[1-\varphi] \varphi+1}-c b\right) \varphi(\varphi-1), \quad \text { and } \quad c=\frac{\alpha(2 a-1)}{b}\left[\frac{\gamma}{\gamma[1-a] a+1}\right]
$$

with $\gamma=e^{b}-2+e^{-b}=2(\cosh (b)-1)$. Setting $\alpha=1$ and $a=4 / 5$ we get

$$
\varphi(\xi)=\frac{1}{2}\left[1+\tanh \left(\frac{7}{5 \sqrt{2}} \xi\right)\right], \quad c=\frac{3 \sqrt{2}}{14} \frac{25 \gamma}{4 \gamma+25}, \quad \gamma=2\left[\cosh \left(\frac{14}{5 \sqrt{2}}\right)-1\right]
$$

as the exact solution to

$$
-c \varphi^{\prime}(\xi)=\varphi(\xi+1)-2 \varphi(\xi)+\varphi(\xi-1)-\left(\frac{\gamma(2 \varphi-1)}{\gamma[1-\varphi] \varphi+1}-\frac{3}{5} \frac{25 \gamma}{4 \gamma+25}\right) \varphi(\varphi-1) .
$$

This solution is illustrated in Figure 5(c) and $f$ is illustrated in Figure 6. In Table 1 we summarize the results of numerical experiments obtained by varying $k$, the number of collocation points per subinterval, $T=T_{+},-T_{-}$that defines the finite interval, and TOL the tolerance on the three components $\varphi, \varphi^{\prime}$, and $c$. We employ the classical phase condition $\varphi(0)=1 / 2$ and report on $N$, the number of mesh points that were employed, $h_{\max }$ and $h_{\min }$, the maximum and minimum subinterval lengths, respectively, and three measures of the error $E_{0}, E_{1}$, and $\operatorname{Err}_{c}$. Error $E_{0}$ is the maximum error between the computed and exact solution obtained by computing at all the mesh points; similarly $E_{1}$ is the error in the first derivative, and $E r r_{c}$ is the error in the wavespeed $c$. The numerical results in Table 1 are not sufficient to establish convergence in the collocation error or in the convergence as a function of the length of the finite interval $T$. The results show good proportionality to the tolerance, with the error dominated by error in the first derivative.

3.4. Ising model. Next we consider traveling wave solutions of an Ising model with convolution operator. Our original equations are of the form (see [16] for the Glauber type Ising model and [20] for the nonsymmetric logarithmic nonlinearity; see also [8])

$$
\dot{v}_{i}+v_{i}=\tanh \left(\frac{\beta}{2 d_{1} d_{2}}(J * v)_{i}-\frac{1}{4 d_{2}}\left(b-v_{i}\right)-\frac{1}{2} \ln \left(\frac{1-b}{1+b}\right)\right), \quad i \in \mathbb{Z},
$$

where $\beta>0, d_{1}>0,0<d_{2}<\frac{1-b^{2}}{4},-1<b<+1,(J * v)_{i}=\sum_{j=1}^{\infty} \alpha_{j}\left(v_{i+j}+v_{i-j}\right)$, and $\sum_{j=1}^{\infty} \alpha_{j}=1$. Applying the traveling wave ansatz $\varphi(i-c t)=v_{i}(t)$ to $(3.9)$ we obtain

$$
-c \varphi^{\prime}(\xi)+\varphi(\xi)=\tanh \left(\frac{\beta}{2 d_{1} d_{2}}(\tilde{J} * \varphi)(\xi)-\frac{1}{4 d_{2}}(b-\varphi(\xi))-\frac{1}{2} \ln \left(\frac{1-b}{1+b}\right)\right), \quad \xi \in \mathbb{R}
$$

where $(\tilde{J} * \varphi)(\xi)=\sum_{j=1}^{\infty} \alpha_{j}(\varphi(\xi+j)+\varphi(\xi-j))$. 
(a)

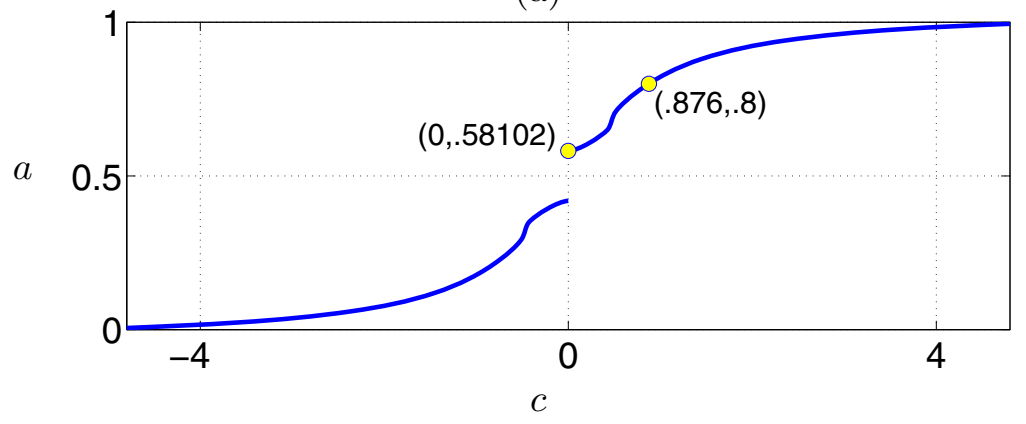

(b)

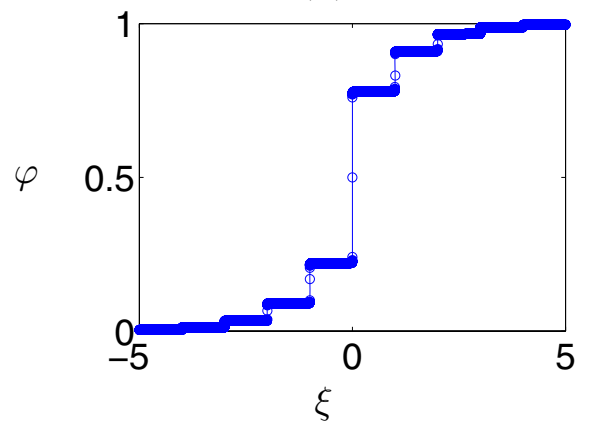

(c)

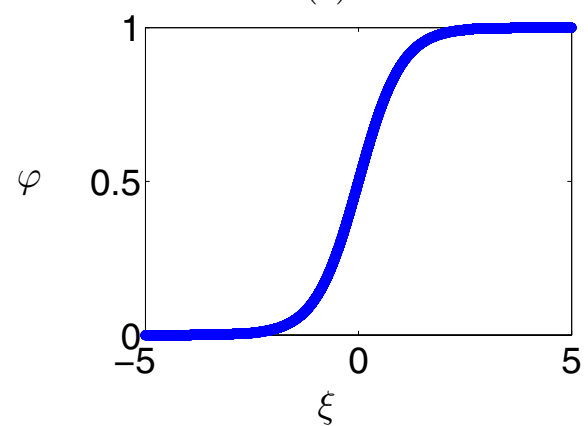

Figure 5. The a(c) curve, (a), and two example wave forms, (b), where $a=.58102$, and (c), where $a=.8$. The value of $\alpha=1$ and the intervals of a where $g$ are nonzero are approximately $[0.20949172139469,0.41898344278938] \cup[0.58101655721062,0.79050827860531]$.

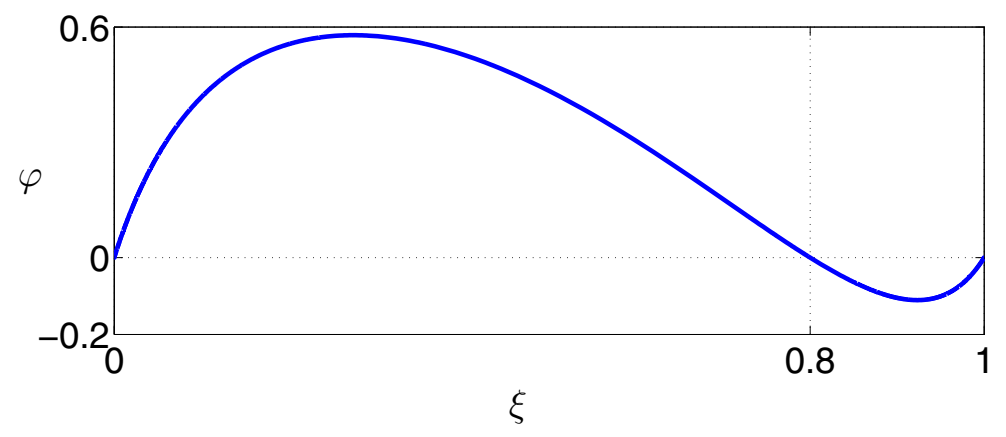

Figure 6. The function $f(\varphi)$ for $\alpha=1$ and $a=.8$.

After normalizing, grouping, and renaming some parameters we consider the equation

$$
-c \varphi^{\prime}(\xi)+\varphi(\xi)=\tanh \left((\tilde{J} * \varphi)(\xi)+\alpha_{0} \varphi(\xi)+\beta\right), \quad \xi \in \mathbb{R},
$$

where $\alpha_{0}>0$ and $\beta \in \mathbb{R}$. We wish to find connecting orbits between two homogeneous equilibria. A homogeneous equilibrium, $z$, satisfies

$$
\frac{1}{2} \ln \left(\frac{1+z}{1-z}\right)=\left(2+\alpha_{0}\right) z+\beta .
$$


Table 1

\begin{tabular}{|c|c|c|c|c|c|c|c|c|}
\hline$k$ & $T$ & TOL & $N$ & $h_{\max }$ & $h_{\min }$ & $E_{0}$ & $E_{1}$ & $E_{r} r_{c}$ \\
\hline 3 & 10 & $1 \mathrm{E}-4$ & 35 & 1.24 & 0.26 & $1.4 \mathrm{E}-7$ & $3.9 \mathrm{E}-5$ & $1.7 \mathrm{E}-7$ \\
\hline 6 & 10 & $1 \mathrm{E}-4$ & 11 & 2.77 & 1.59 & $2.4 \mathrm{E}-5$ & $1.8 \mathrm{E}-3$ & $1.8 \mathrm{E}-4$ \\
\hline 3 & 10 & $1 \mathrm{E}-6$ & 77 & 1.27 & 0.07 & $2.9 \mathrm{E}-9$ & $3.8 \mathrm{E}-7$ & $4.2 \mathrm{E}-11$ \\
\hline 6 & 10 & $1 \mathrm{E}-6$ & 19 & 2.00 & 0.64 & $1.5 \mathrm{E}-8$ & $9.9 \mathrm{E}-6$ & $3.0 \mathrm{E}-8$ \\
\hline 3 & 40 & $1 \mathrm{E}-4$ & 57 & 3.65 & 0.43 & $1.1 \mathrm{E}-6$ & $6.3 \mathrm{E}-4$ & $1.3 \mathrm{E}-6$ \\
\hline 6 & 40 & $1 \mathrm{E}-4$ & 29 & 3.63 & 2.22 & $1.8 \mathrm{E}-5$ & $5.7 \mathrm{E}-3$ & $2.3 \mathrm{E}-4$ \\
\hline 3 & 40 & $1 \mathrm{E}-6$ & 97 & 5.89 & 0.09 & $2.6 \mathrm{E}-9$ & $6.4 \mathrm{E}-7$ & $3.0 \mathrm{E}-10$ \\
\hline 6 & 40 & $1 \mathrm{E}-6$ & 61 & 2.41 & 0.71 & $2.3 \mathrm{E}-8$ & $1.6 \mathrm{E}-5$ & $5.2 \mathrm{E}-8$ \\
\hline
\end{tabular}

Thus, a necessary condition for the existence of one positive and one negative homogeneous equilibrium solution is that $|\beta|<\left(2+\alpha_{0}\right)$. We can guarantee the existence of such equilibria by taking $\alpha_{0}$ sufficiently large. For $\beta>0$, the existence of one negative homogeneous equilibria typically implies the existence of two negative homogeneous equilibria, and similarly for $\beta<0$.

Next consider the linearization about an arbitrary homogeneous equilibrium solution, $z$. We focus on the case where $\alpha_{1}=1$ and $\alpha_{j}=0$ for $j=2,3, \ldots$ The characteristic function is given by

$$
h(\lambda)=c \lambda-1+\alpha_{0} \gamma+2 \gamma \cosh (\lambda)
$$

where $\gamma=\operatorname{sech}^{2}\left(\left(2+\alpha_{0}\right) z+\beta\right)$, so $0 \leqslant \gamma \leqslant 1$. So, to have one positive and one negative real eigenvalue we must have $2+\alpha_{0}<1 / \gamma$. Thus, a necessary condition for a connecting orbit between one positive, $z_{+}$, and one negative, $z_{-}$, homogeneous equilibria is

$$
|\beta|<2+\alpha_{0}<1 / \bar{\gamma}
$$

where $\bar{\gamma}=\max \left\{\gamma_{-}, \gamma_{+}\right\}$and $\gamma_{\bullet}=\operatorname{sech}^{2}\left(\left(2+\alpha_{0}\right) z_{\bullet}+\beta\right)$ for $\bullet=+$ or - .

The Fredholm theory of Mallet-Paret $[29,30]$ is applicable to (3.11) and shows the existence of monotone solutions and a monotone $(\beta, c)$ curve for $c \neq 0$. The condition (ii) on page 56 of [30] requires that $\alpha_{j} \geqslant 0$ for all $j$, while our condition (3.14) implies condition (v) on page 56 of [30]. In Figure 7 we show the results of some of our numerical experiments. Figure 7 is a plot of the function in (3.12) and a plot of a $(\beta, c)$ curve, the analogue for this problem of an $(a, c)$ curve.

3.5. Frenkel-Kontorova type equations. Consider the FK type equation [40, 41]

$$
\begin{aligned}
\ddot{x}_{j}+\gamma \dot{x}_{j} & =x_{j-1}-2 x_{j}+x_{j+1}-d \sin x_{j}+F, \quad j=1, \ldots, N, \\
x_{j+N} & =x_{j}+2 \pi M .
\end{aligned}
$$

Here the $x_{j}$ 's denote the positions of particles in a chain, $N$ is the number of particles, $F$ is an applied force, and $M$ is an arbitrary integer. This equation was first proposed to describe the motion of dislocations in crystals but is now used to model a number of processes. The equation admits traveling wave solutions, which are referred to as uniform sliding states in the physics literature. 

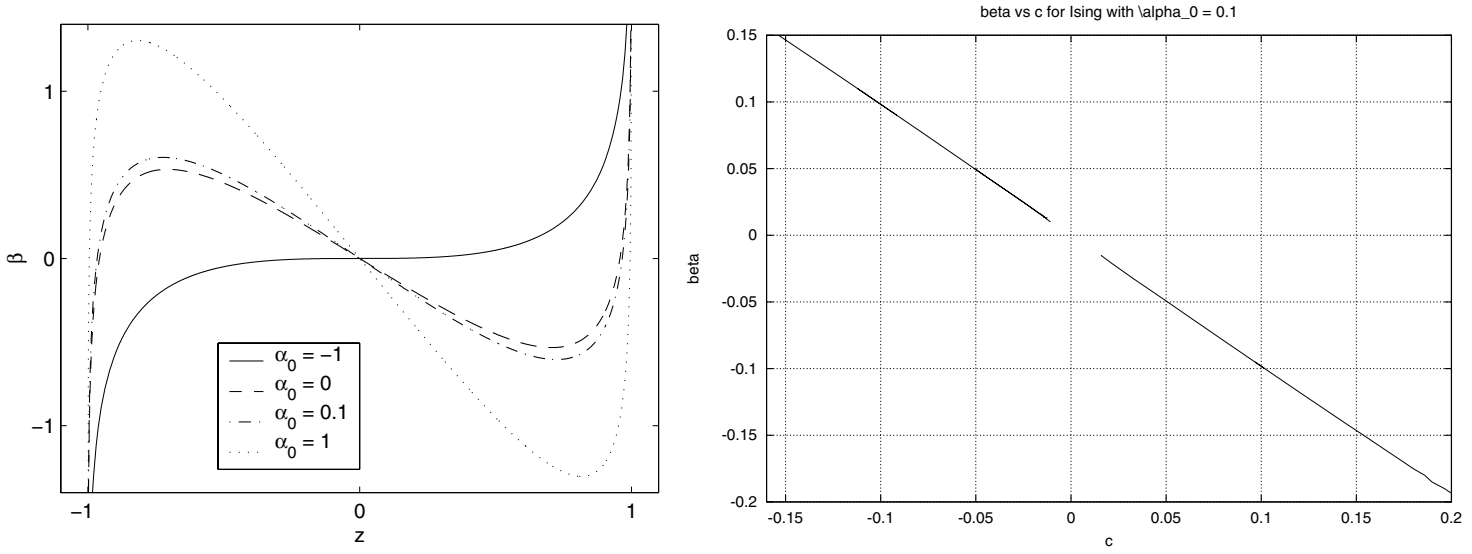

Figure 7. (i) Plots of $0.5 \ln ((1+z) /(1-z))-2-\alpha_{0}=0$ for different values of $\alpha_{0}$. The roots of (3.12) may thus be determined by intersection with a horizontal line corresponding to values of $\beta$. (ii) $A(\beta, c)$ curve for the Ising model with $\alpha_{0}=0.1$. become

Using the traveling wave ansatz $x_{j}(t)=\varphi(\sigma j-c t)$, where $\sigma=\frac{2 \pi M}{N},(3.15)$ and (3.16)

$$
\begin{aligned}
c^{2} \ddot{\varphi}(\xi)-\gamma c \dot{\varphi}(\xi) & =L_{\sigma} \varphi(\xi)-d_{1} \sin (\varphi(\xi))+F, \\
\varphi(\xi+2 \pi) & =\varphi(\xi)+2 \pi M,
\end{aligned}
$$

where

$$
L_{\sigma} \varphi(\xi)=\varphi(\xi-\sigma)-2 \varphi(\xi)+\varphi(\xi+\sigma) \text { and } \quad \xi=\sigma j-c t
$$

The particular choice of $\sigma$ is made in order to achieve a simple relationship between the velocity $c$ of the traveling wave and the average particle velocity. The boundary conditions (3.16) imply

$$
\begin{aligned}
x_{j}\left(t-\frac{2 \pi M}{c}\right) & =\varphi\left(\sigma j-c\left[t-\frac{2 \pi M}{c}\right]\right)=\varphi(\sigma j+2 \pi M-c t) \\
& =\varphi(\sigma j-c t)+2 \pi M=x_{j}(t)+2 \pi M .
\end{aligned}
$$

Thus the average particle velocity is $-c$, the negative of the traveling wave velocity.

Because of the discrete translational symmetry in (3.17) it is sufficient to solve (3.17) subject to $\varphi(\xi+2 \pi)=\varphi(\xi)+2 \pi$ to obtain solutions to (3.17), (3.18). We choose to solve (3.17) on the interval $[-\pi, \pi]$ subject to the conditions

$$
\varphi(\pi)=\varphi(-\pi)+2 \pi \quad \text { and } \quad \varphi(0)=0,
$$

where $\varphi(0)=0$ is a phase condition and specifies a unique phase when $\varphi$ is monotonic. In the case of nonmonotonic $\varphi$ (as will arise in this example), this condition no longer guarantees a unique solution to the equations, but in general solutions will be locally unique, a sufficient condition for the numerical convergence. 
An alternative formulation of this problem is

$$
\left\{\begin{aligned}
c^{2} \ddot{\psi}(\xi)-\gamma c[1+\dot{\psi}(\xi)] & =L_{\sigma} \psi(\xi)-d_{1} \sin (\xi+\psi(\xi))+F \\
\psi(\pi) & =\psi(-\pi), \\
\psi(0) & =0
\end{aligned}\right.
$$

with

$$
\varphi(\xi)=\xi+\psi(\xi)
$$

The function $\psi$, referred to as the dynamic hull function, is periodic.

We numerically solve both formulations, where we choose $F$ and solve the auxiliary equation $\dot{c}=0$ to find $c$, as in previous examples. One can also fix $c$ and solve an auxiliary equation $\dot{F}=0$ to find $F$, which is often easier.

In the linear case where $d_{1}=0$ it is simple to verify that $\varphi(\xi)=\xi$ and $\psi(\xi)=0$ solve (3.17), (3.19), and (3.20), respectively, with $F=-\gamma c$.

Following [40] in Figure 8 we present computations for $\gamma=0.5, M=89, N=233$, and $d_{1}=1,2$, and 3 . There is good agreement between the velocity-force characteristics in Figure 8(i) and those in Figure 2 of [40], which were computed by a different method. The cusps seen in Figure 8(i) are caused by resonances.
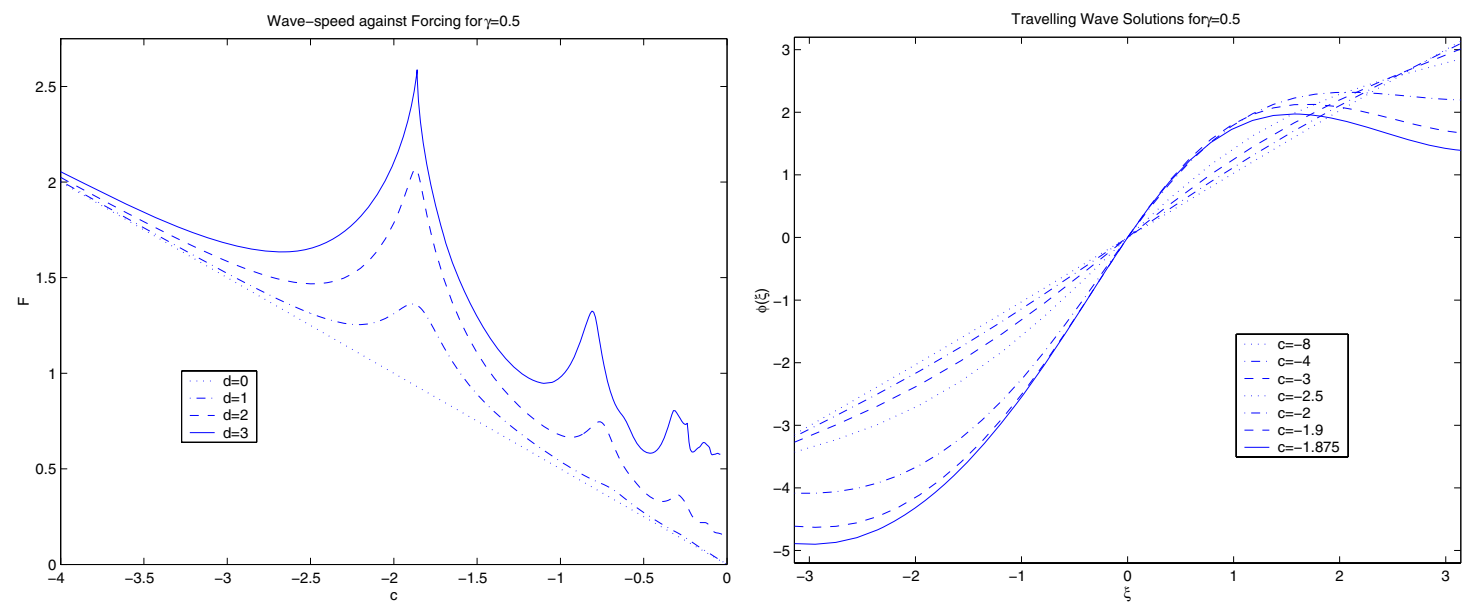

Figure 8. Solutions of (3.17), (3.19) with $\gamma=0.5, M=89, N=233$. (i) Traveling wave velocity c against applied force $F$ for $d_{1}=1,2$, and 3. (ii) Traveling wave profiles for $d=2$.

For $-c$ large, above the first resonance, all the graphs are close to the line $F=-\gamma c$ which corresponds to the linear case $d_{1}=0$. As in the previous examples, propagation failure is numerically resolvable depending on the parameter $d_{1}$, with an interval of propagation failure becoming obvious for $d_{1}$ large $\left(d_{1} \geqslant 2\right)$.

In Figure 8(ii) we show the evolution of the traveling wave profile as $-c$ is decreased toward the first resonant velocity. For large velocities, motion is essentially linear, but as the traveling wave velocity is decreased it becomes less so, and it becomes nonmonotonic at the first turning point of the graph at $-c \approx 2.5$. 
For $d_{1}=2$ and $F \in[1.5,2]$ it is clear from Figure $8(\mathrm{i})$ that there exist traveling waves with three different wavespeeds. We find numerically that only the wave of largest speed is monotonic. In Figure 9(i) we show the three traveling waves for $F=1.75$.
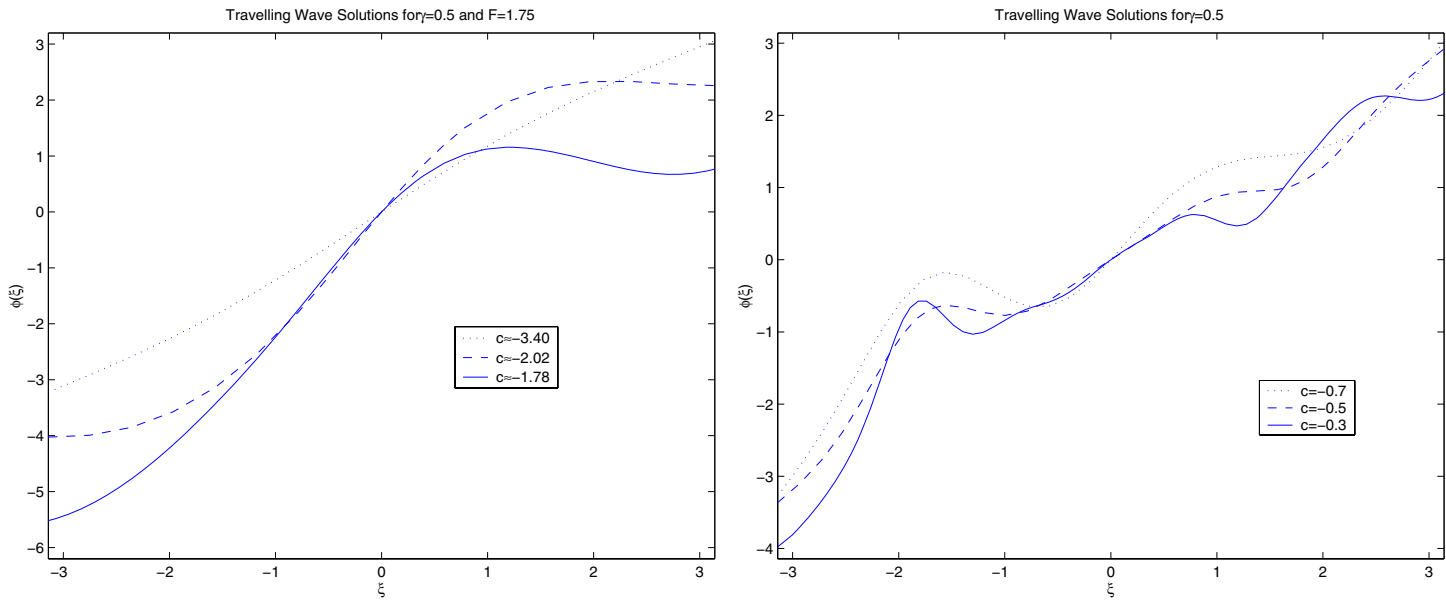

Figure 9. Solutions of (3.17), (3.19) with $\gamma=0.5, M=89, N=233$, and $d_{1}=2$. (i) Three traveling waves for $F=1.75$. (ii) Traveling waves for $c=-0.7, c=-0.5, c=-0.3$.

As the wavespeed decreases the form of the traveling wave solution becomes progressively more complicated as demonstrated in Figure 9(ii).
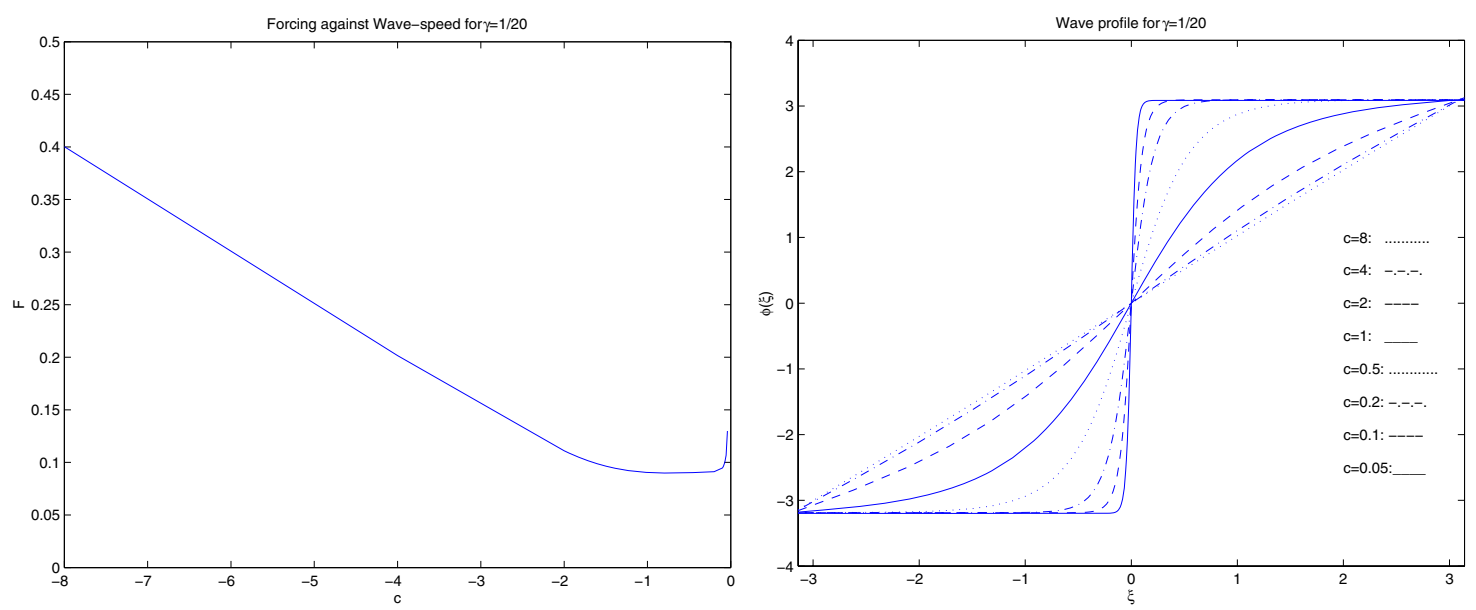

Figure 10. Solutions of (3.17), (3.19) with $\gamma=1 / 20, M=1, N=200$, and $d_{1}=2$. (i) Applied force $F$ against traveling wave velocity $c$. (ii) Traveling wave profiles.

Consider briefly the case $M=1, N=200, d_{1}=2$, and $\gamma=1 / 20$. For this case the first resonant velocity is very small at approximately $\pi / 100$. Figure 10 (i) shows the $F$ against $c$ profile to the first resonant velocity. Figure 10(ii) shows corresponding traveling wave profiles. For $M / N \ll 1$ we obtain step-like traveling wave solutions, much like the behavior seen in the other examples (with the exception that the $F(c)$ curve again fails to be monotonic). 
4. Numerical analysis. In this section we describe the implementation of our methods in the software package COLMTFDE (COLlocation for Mixed Type Functional Differential Equations) we are developing, which was used to produce the results presented above, and the numerical analysis issues which arise. COLMTFDE is a collocation boundary value problem solver and is a member of the COLSYS family $[2,6,13,42]$. Thus we pay particular attention to the differences between our code and other members of the COLSYS family. The main difference is the ability to handle delayed (and advanced) terms directly. The size of the delays $\left\{s_{\sigma}(x)\right\}_{\sigma=1}^{n}$ and even their number $n$ may be dependent on $x . F_{I}$ in (1.1) can be a fully nonlinear function of $u_{I}\left(x+s_{\sigma}\right)$ and its derivatives up to and including the $m$ th derivative (which we made use of in section 3.4). Also of significance is COLMTFDE's ability to handle implicitly defined boundary functions, as described in section 2.3, as well as explicitly defined boundary functions. COLMTFDE also allows nonseparated boundary conditions of the form

$$
\sum_{J=1}^{m^{*}} \mathcal{B}_{J} y(\zeta(J))=\beta_{J},
$$

whereas most of the COLSYS family of boundary value problem solvers require that the boundary conditions for the problem are separated.

The introduction of the scalar factors $\tau_{I}(x)$ multiplying the highest order term on the left-hand side of (1.1) also constitutes a significant generalization from other members of the COLSYS family, further extending the range of problems which may be solved. This is particularly important in problems such as the spatially discrete Nagumo equation where a traveling wave with speed $c$ satisfies (3.4). Two boundary conditions are needed to compute a numerical solution due to the presence of the second-order difference term $(\varphi(\xi+1)-2 \varphi(\xi)+\varphi(\xi-1))$ on the right-hand side of (3.4). However, since there is only a first-order derivative term present, a boundary value problem solver will only expect and indeed allow one boundary value, which will result in a singular linear system. This difficulty is resolved by introducing a second-order derivative term $\tau \ddot{\varphi}(\xi)$ as in (3.4) and then solving the problem with $\tau=0$, as in Figure 3 .

Other members of the COLSYS family use local parameter condensation to eliminate variables which are internal to subintervals of the mesh, thus simplifying the linear system and reducing storage requirements. They also take advantage of the almost-block-diagonal nature of the resulting linear matrix to solve this system extremely efficiently.

However, parameter condensation is not practical for a functional differential equation solver, as the forward and backward delay terms typically fall between collocation points in different mesh intervals, and hence the so-called local variables are no longer truly local. Also, these delay terms will not usually fall on the sub- or superdiagonals of the matrix, and their relative locations will therefore not only depend on the size of the mesh but will require updating with each mesh refinement. The resulting linear matrix will therefore not in general be almost-block-diagonal.

COLMTFDE therefore cannot make use of parameter condensation or block-diagonal structure. This has significant computational costs, as construction and storage of the full linear matrix, which has size $\left(N\left(m^{*}+k d\right)+m^{*}\right)^{2}$, requires considerably more memory than the condensed matrix, of size $N\left(2 m^{* 2}+3 m^{*} k d+k d^{2}\right)$, stored by COLMOD. In addition, since the matrix is no longer sparse almost-block-diagonal we cannot take advantage of efficient 
solvers for such systems, and use a full forward-backward substitution method. The loss of sparseness cannot be avoided in general, as problems such as the Ising problem of section 3.5 will always result in dense matrices.

4.1. Collocation formulation. We consider a $k$-stage collocation scheme for (1.1). Let

$$
\pi: T_{-}=x_{0}<x_{1}<\cdots<x_{N-1}<x_{N}=T_{+},
$$

$h_{i}:=x_{i+1}-x_{i}$, and $h:=\max _{i=0, \ldots, N}\left\{h_{i}\right\}$. Let $\left\{\rho_{i}\right\}_{i=1}^{k}$ denote $k$ Gaussian collocation points ordered so that

$$
0 \leqslant \rho_{1}<\cdots<\rho_{k} \leqslant 1
$$

We seek a collocation solution

$$
\mathbf{u}^{\pi}(x)=\left(u_{1}^{\pi}, u_{2}^{\pi}, \ldots, u_{d}^{\pi}\right)^{T},
$$

such that for each $I=1, \ldots, d$, the $I$ th component $u_{I}^{\pi}(x)$ is a piecewise polynomial function such that $u_{i}^{\pi} \in \mathcal{P}_{k+m_{I}, \pi} \cap \mathcal{C}^{m_{I}-1}\left(T_{-}, T_{+}\right)$, where $\mathcal{P}_{k+m_{I}, \pi}$ is the space of functions which reduce to polynomials of order $k+m_{I}$ on each of the subintervals $\left[x_{i}, x_{i+1}\right]$ of $\pi$, for some $k \geqslant \max _{I=1, d} m_{I}$. The differential equation is satisfied at the $k N$ collocation points

$$
x_{i j}=x_{i}+h_{i} \rho_{j}, \quad j=1, \ldots, k, \quad i=1, \ldots, N .
$$

Note that since $\mathcal{P}_{k+m_{I}, \pi} \cap \mathcal{C}^{m_{I}-1}\left(T_{-}, T_{+}\right)$is of dimension $k N+m_{I}$ and there are $k N$ collocation conditions associated with the $I$ th equation, there must be $m_{I}$ side conditions resulting from this equation and thus $m^{*}$ side conditions in total. This exactly matches the number of boundary conditions in (1.1), which $\mathbf{u}^{\pi}$ is thus required to satisfy.

We employ the now standard monomial Runge-Kutta basis representation. Consider a fixed mesh element $x \in\left[x_{i}, x_{i+1}\right]$. Then, writing $u$ for an arbitrary component $u_{I}$ and $m$ for $m_{I}, I \in 1, \ldots, d$, each polynomial $u^{\pi}(x)$ can be expressed in terms of its Taylor series about $x_{i}$ as

$$
u^{\pi}(x)=\sum_{j=1}^{k+m} \frac{\left(x-x_{i}\right)^{j-1}}{(j-1) !} \mathcal{D}^{j-1} u_{\pi}\left(x_{i}\right)=\sum_{j=1}^{m} \frac{\left(x-x_{i}\right)^{j-1}}{(j-1) !} y_{i j}+h_{i}^{m} \sum_{j=1}^{k} \psi_{m j}\left(\frac{x-x_{i}}{h_{i}}\right) z_{i j}
$$

where

$$
\begin{array}{ll}
\mathcal{D}^{l-1} \psi_{m j}(0)=0, & 1 \leqslant l \leqslant m, \quad 1 \leqslant j \leqslant k, \\
\mathcal{D}^{m} \psi_{m j}\left(\rho_{l}\right)=\delta_{j l}, & 1 \leqslant l \leqslant k .
\end{array}
$$

Hence, corresponding to (4.2),

$$
y_{i j}=\mathcal{D}^{j-1} u^{\pi}\left(x_{i}\right), \quad 1 \leqslant j \leqslant m, \quad \text { and } \quad z_{i j}=\mathcal{D}^{m} u^{\pi}\left(x_{i j}\right), \quad 1 \leqslant j \leqslant k,
$$

and so the collocation solution, $\mathbf{u}^{\pi}$, can now be written in terms of

$$
\mathbf{y}_{i}:=\left(y_{i 1}, \ldots, y_{i m}\right)^{T}, \quad \mathbf{z}_{i}:=\left(z_{i 1}, \ldots, z_{i k}\right)^{T},
$$

and approximating the solution of (1.1) is reduced to finding these vectors. However even in the linear case the details of the construction of this system are somewhat complicated, and we describe them below. 
4.1.1. Linear systems. The functions $F_{I}$ and $G_{J}$ in (1.1) are usually nonlinear. When they are linear, the system can be written in the form

$$
\gamma_{I} \mathcal{D}^{\left(m_{I}\right)} u_{I}(x)=\sum_{t=1}^{d}\left[\sum_{l=1}^{m_{t}} c_{t l}^{I}(x) D^{(l-1)} u_{t}(x)+\sum_{l=1}^{m_{t}+1} \boldsymbol{\alpha}_{t l}^{I}(x) D^{(l-1)} \overline{\mathbf{u}}_{t}(x)\right]+q_{I}(x)
$$

with $I=1, \ldots, d, x \in\left(T_{-}, T_{+}\right)$,

$$
\boldsymbol{\alpha}_{t l}^{I}(x):=\left(\alpha_{t l}^{I}\left(x+s_{1}(x)\right), \ldots, \alpha_{t l}^{I}\left(x+s_{n}(x)\right)\right)^{T},
$$

and boundary conditions as in (1.1).

The process described above leads to a linear system of equations for $\mathbf{u}^{\pi}$ and its first $(m-1)$ derivatives. We now outline the construction of this system, first for $d=1$, and then in the more complicated higher-dimensional case.

4.1.2. One-dimensional system. Write the one-dimensional linear delay system as

$$
\tau \mathcal{D}^{m} u(x)=\sum_{l=1}^{m} c_{l}(x) D^{(l-1)} u(x)+\sum_{\sigma=1}^{n}\left[\sum_{l=1}^{m+1} \alpha_{l}\left(x+s_{\sigma}\right) D^{(l-1)} u\left(x+s_{\sigma}\right)\right]+q(x),
$$

and suppose that for some delay $s_{\sigma}$, where $\sigma \in\{1, \ldots, n\}$, and some collocation point $x_{i r}$, $i \in\{1, \ldots, N\}, r \in\{1, \ldots, k\}$, we have $x_{\omega} \leqslant x_{i r}+s_{\sigma}<x_{\omega+1}$; i.e., we identify the mesh interval $\left[x_{\omega}, x_{\omega+1}\right]$ in which the delayed collocation point lies. For convenience, we denote $x_{r \sigma}^{i}:=x_{i r}+s_{\sigma}$; then, from (4.2), we have the following representation for the function $u^{\pi}$ and its derivatives at the point $x_{r \sigma}^{i}$ :

$$
\begin{aligned}
D^{(l-1)} u_{\pi}\left(x_{r \sigma}^{i}\right)= & \sum_{j=l}^{m} \frac{\left(x_{r \sigma}^{i}-x_{\omega}\right)^{j-l}}{(j-l) !} y_{\omega j}+h_{\omega}^{m-l+1} \sum_{j=1}^{k} D^{(l-1)} \psi_{m j}\left(\frac{x_{r \sigma}^{i}-x_{\omega}}{h_{\omega}}\right) z_{\omega j}, \\
& D^{m} u\left(x_{r \sigma}^{i}\right)=h_{\omega} \sum_{j=1}^{k} D^{m} \psi_{m j}\left(\frac{x_{r \sigma}^{i}-x_{\omega}}{h_{\omega}}\right) z_{\omega j} .
\end{aligned}
$$

Since $u^{\pi}$ must satisfy (4.4) at each collocation point $x_{i j}$, applying the collocation equations yields the $k$ equations for $\mathbf{y}_{i}$ and $\mathbf{z}_{i}$,

$$
-\left(\mathcal{V}_{i}+\sum_{\sigma=1}^{n} \mathcal{P}_{i}^{\sigma}\right) \mathbf{y}_{i}+\left(\mathcal{W}_{i}-\sum_{\sigma=1}^{n} \mathcal{Q}_{i}^{\sigma}\right) \mathbf{z}_{i}=\mathbf{q}_{i}, \quad 1 \leqslant i \leqslant N
$$

where $\mathcal{V}_{i}$ and $\mathcal{P}_{i}$ are $k \times m$ matrices with entries

$$
\mathcal{V}_{r j}^{i}=\sum_{l=1}^{j} \frac{c_{l}\left(x_{i r}\right)\left(h_{i} \rho_{r}\right)^{j-l}}{(j-l) !}, \quad \mathcal{P}_{r j}^{i}=\sum_{l=1}^{j} \frac{\alpha_{l}\left(x_{r \sigma}^{i}\right)\left(x_{r \sigma}^{i}-x_{\omega}\right)^{j-l}}{(j-l) !}, \quad 1 \leqslant r \leqslant k, \quad 1 \leqslant j \leqslant m
$$


and $\mathcal{W}_{i}$ and $\mathcal{Q}_{i}$ are $k \times k$ matrices with entries

$$
\left.\begin{array}{c}
\mathcal{W}_{r j}^{i}=\tau \delta_{r j}-\sum_{l=1}^{m} c_{l}\left(x_{i r}\right) h_{i}^{m+1-l} D^{(l-1)} \psi_{m j}\left(\rho_{r}\right), \\
\mathcal{Q}_{r j}^{i}=\sum_{l=1}^{m+1} \alpha_{l}\left(x_{r \sigma}^{i}\right) h_{i}^{m+1-l} D^{(l-1)} \psi_{m j}\left(\frac{x_{r \sigma}^{i}-x_{\omega}}{h_{\omega}}\right),
\end{array}\right\} 1 \leqslant r, j \leqslant k .
$$

We let $\mathbf{q}_{i}:=\left(q\left(x_{i 1}\right), \ldots, q\left(x_{i k}\right)\right)^{T}$ and recall that $\mathbf{y}_{i}$ and $\mathbf{z}_{i}$ were defined in (4.3).

From the $m$ global continuity requirements, we obtain the additional relations

$$
\mathcal{C}_{i} \mathbf{y}_{i}+\mathcal{D}_{i} \mathbf{z}_{i}=\mathbf{y}_{i+1}, \quad 1 \leqslant i \leqslant N,
$$

where $\mathcal{C}$ is an $m \times m$ matrix with entries

$$
\mathcal{C}_{r j}^{i}=\frac{h_{i}^{j-r}}{(j-r) !}, \quad j \geqslant r
$$

and $\mathcal{D}$ is of order $m \times k$ with entries

$$
\mathcal{D}_{r j}^{i}=h_{i}^{m+1-r} D^{(r-1)} \psi_{m j}(1), \quad 1 \leqslant r \leqslant m, \quad 1 \leqslant j \leqslant k .
$$

We therefore have a linear system for $u^{\pi}$ and its first $m-1$ derivatives.

4.1.3. Higher-dimensional system. For systems of equations, the situation is more complicated, and the notation becomes rather convoluted. However, it is worth giving in detail, as the construction of the linear matrix for a mixed system is not immediately obvious.

Applying the collocation equations for a system with $d>1$ yields $k d$ equations for $\mathbf{y}_{i}$ and $\mathbf{z}_{i}$,

$$
-\left(\overline{\mathcal{V}}_{i}+\sum_{\sigma=1}^{n} \overline{\mathcal{P}}_{i}^{\sigma}\right) \mathbf{y}_{i}+\left(\overline{\mathcal{W}}_{i}-\sum_{\sigma=1}^{n} \overline{\mathcal{Q}}_{i}^{\sigma}\right) \mathbf{z}_{i}=\mathbf{q}_{i}, \quad 1 \leqslant i \leqslant N
$$

where $\overline{\mathcal{V}}_{i}$ is a $k d \times m^{*}$ matrix, whose entries are themselves matrices, so

$$
\overline{\mathcal{V}}_{R J}^{i}=\left[V^{i}\right], \quad 1 \leqslant R \leqslant k, \quad 1 \leqslant J \leqslant d,
$$

where $V^{i}$ is a $d \times m_{J}$ matrix with entries

$$
V_{I j}^{i}=\sum_{l=1}^{j} \frac{c_{J l}^{I}\left(x_{i R}\right)\left(h_{i} \rho_{R}\right)^{j-l}}{(j-l) !}, \quad 1 \leqslant I \leqslant d, \quad 1 \leqslant j \leqslant m_{J} .
$$

In a similar fashion, $\overline{\mathcal{W}}_{i}$ is a $k d \times k d$ matrix, with entries again consisting of submatrices,

$$
\begin{gathered}
\overline{\mathcal{W}}_{R T}^{i}=\left[W^{i}\right], \quad 1 \leqslant R, T \leqslant k \\
W_{I j}^{i}=\tau_{I} \delta_{R T} \delta_{I j}-\sum_{l=1}^{m_{j}} c_{j l}^{I}\left(x_{i R}\right) h_{i}^{m_{j}+1-l} D^{(l-1)} \psi_{m_{j} T}\left(\rho_{R}\right), \quad 1 \leqslant I, j \leqslant d .
\end{gathered}
$$


Also, $\overline{\mathcal{P}}_{i}^{\sigma}$ is a $k d \times m^{*}$ matrix with $d \times m_{J}$ matrix subentries

$$
\overline{\mathcal{P}}_{R J}^{i \sigma}=\left[P^{i \sigma}\right], \quad \begin{aligned}
& 1 \leqslant R \leqslant k, \\
& 1 \leqslant J \leqslant d,
\end{aligned} \quad \text { where } \quad P_{I j}^{i \sigma}=\sum_{l=1}^{j} \frac{\alpha_{J l}^{I}\left(x_{R \sigma}^{i}-x_{\omega}\right)^{j-l}}{(j-1) !}, \quad \begin{gathered}
1 \leqslant I \leqslant d, \\
1 \leqslant j \leqslant m_{J},
\end{gathered}
$$

and $\overline{\mathcal{Q}}_{i}^{\sigma}$ is a $k d \times k d$ matrix with entries again consisting of submatrices

$$
\begin{gathered}
\overline{\mathcal{Q}}_{R T}^{i \sigma}=\left[Q^{i \sigma}\right], \quad 1 \leqslant R, T \leqslant k, \\
Q_{I j}^{i \sigma}=\sum_{l=1}^{m_{j}+1} \alpha_{j l}^{I}\left(x_{r \sigma}^{i}\right) h_{i}^{m_{j}+1-l} D^{(l-1)} \psi_{m_{j} T}\left(\frac{x_{R \sigma}^{i}-x_{\omega}}{h_{\omega}}\right), \quad 1 \leqslant I, j \leqslant d .
\end{gathered}
$$

From the $m^{*}$ boundary conditions, we obtain $m^{*}$ additional relations,

$$
\overline{\mathcal{C}}_{i} \mathbf{y}_{i}+\overline{\mathcal{D}}_{i} \mathbf{z}_{i}=\mathbf{y}_{i+1}, \quad 1 \leqslant i \leqslant N,
$$

where $\overline{\mathcal{C}}$ is an $m^{*} \times m^{*}$ matrix with matrix entries

$$
\overline{\mathcal{C}}_{I S}^{i}=\delta_{I S}\left[C^{i}\right], \quad 1 \leqslant I, S \leqslant d, \quad \text { where } \quad C_{r j}^{i}=\frac{h_{i}^{j-r}}{(j-r) !}, \quad 1 \leqslant r \leqslant m_{I}, \quad 1 \leqslant j \leqslant m_{S} .
$$

We have that $\overline{\mathcal{D}}$ is of order $m^{*} \times k d$ with entries

$$
\overline{\mathcal{D}}_{I J}^{i}=\delta_{I S}\left[D^{i}\right], \quad \begin{aligned}
& 1 \leqslant I \leqslant d, \\
& 1 \leqslant J \leqslant k,
\end{aligned} \quad \text { with } \quad D_{r s}^{i}=h_{i}^{m_{I}+1-r} D^{(r-1)} \psi_{m_{I} J}(1), \quad \begin{gathered}
1 \leqslant r \leqslant m_{I}, \\
1 \leqslant s \leqslant d .
\end{gathered}
$$

The resulting linear systems are of the form

$$
\left[\begin{array}{cccccccccc}
-V_{1} & -W_{1} & 0 & \cdots & -P_{1}^{1} & -Q_{1}^{1} & \cdots & \cdots & \cdots & \\
-C_{1} & -D_{1} & I & & & & & & & \\
& & \cdot & \cdot & & & & & & \\
-P_{i}^{1} & -Q_{i}^{1} & \cdots & -V_{i} & -W_{i} & 0 & \cdots & -P_{i}^{n} & -Q_{i}^{n} & \\
& & & -C_{i} & -D_{i} & I & & & & \\
& & & & & & \cdot & \cdot & & \\
\cdots & -P_{N}^{1} & -Q_{N}^{1} & \cdots & -P_{N}^{n} & -Q_{N}^{n} & \cdots & -V_{N} & -W_{N} & 0 \\
B_{1} & & & & & & & -C_{N} & -D_{N} & I \\
& & & & & \cdot & \cdot & \cdot & & B_{m^{*}}
\end{array}\right]\left[\begin{array}{c}
y_{1} \\
z_{1} \\
\cdot \\
\cdot \\
y_{i} \\
z_{i} \\
\cdot \\
\cdot \\
y_{N} \\
z_{N} \\
y_{N+1}
\end{array}\right]=\left[\begin{array}{c}
q_{1} \\
0 \\
\cdot \\
\cdot \\
q_{i} \\
0 \\
\cdot \\
\cdot \\
q_{N} \\
0 \\
\beta
\end{array}\right]
$$

4.1.4. Nonlinear systems. For general nonlinear problems of the form (1.1) our code uses the method of quasilinearization, which is common to all members of the COLSYS family of codes. The changes made to allow for delay terms to be treated directly are to the construction and storage of the linear system. The method of solving the nonlinear problem (1.1) is therefore almost identical to that used for COLMOD [1]. The differences involve the order in which quantities are obtained or constructed due to the backward and forward delay terms. 
4.2. Convergence. Assuming sufficient smoothness of the problem coefficients and of the exact solution (except at a finite number of points which form part of the mesh $\pi$ ), much of the standard stability and approximation theory for ODE boundary value problem collocation solutions presented in [3] holds in the presence of delays, with some important changes.

A basic convergence result for the solution of DDEs of this type by collocation is given in [5], and we reproduce it here. Following [5], in order to keep the notation as simple as possible, the result is given in the case of the first-order equation

$$
\tau u^{\prime}(x)=F(x, u(x), \bar{u}), \quad x \in\left(T_{-}, T_{+}\right),
$$

with $\bar{u}_{I}=\left(u\left(x+s_{1}(x)\right), \ldots, u\left(x+s_{n}(x)\right)\right)^{T}$, and where $\left\{s_{\sigma}(x)\right\}_{\sigma=1}^{n}$ is a finite collection of delays, possibly dependent on $x$. Generalization to systems of higher order such as (1.1) is straightforward.

The mesh $\pi$ is defined on the interval $\left[T_{-}, T_{+}\right]$, such that

$$
\pi: T_{-}=x_{0}<x_{1}<\cdots<x_{N-1}<x_{N}=T_{+}
$$

with $h_{i}:=x_{i+1}-x_{i}$ and $h:=\max _{i} h_{i}$. Then, denoting

$$
\min _{x \in\left[T_{-}, T_{+}\right]} s_{1}(x)=\bar{T}_{-}, \quad \max _{x \in\left[T_{-}, T_{+}\right]} s_{n}(x)=\bar{T}_{+},
$$

a solution $u^{*}$ of (4.5) is in general only a piecewise smooth function for $x \in\left[\bar{T}_{-}, \bar{T}_{+}\right]$, and in fact the solution may be discontinuous at $x=T_{-}$or $x=T_{+}$.

To establish convergence assume the right-hand side $F$ of the DDE (4.5) is sufficiently smooth. Then, since the delayed terms $\left\{s_{\sigma}(x)\right\}_{\sigma=1}^{n}$ depend only on $x$, the locations at which the solution and its derivatives up to order $p$ have potentially nonsmooth behavior can be precomputed. Including all these points in the partition $\pi$ yields

$$
u^{*} \in C_{\pi}^{p}[a, b] \cap C[a, b] .
$$

Note that if more smoothness, or in other words a larger $p$, is required, then the partition $\pi=\pi(p)$ becomes more dense in general. The appropriate choice of $p$ for practical computation depends on the boundary value problem and the required accuracy of the numerical solution.

The following convergence results for $(4.5)$ are due to Bader $[4,5]$.

Theorem 4.1. Let $u^{*} \in C^{p+1}[a, b]$ for $p \geqslant 1$ be a solution of the problem (4.5) and suppose the following:

(i) F is sufficiently smooth.

(ii) The linearized problem associated with $u^{*}$ is uniquely solvable and has a Green's function $H\left(x, x_{i}\right)$.

Then there exist $\delta, \varepsilon$ so that the following hold:

(a) There is no other solution $\tilde{u}$ for $\left|D\left(u^{*}-\tilde{u}\right)\right|<\varepsilon$.

(b) For $h \leqslant \delta$ there is a unique collocation solution $u_{\pi} \in P_{k+1, \pi} \cap C[a, b]$ in this neighborhood of $u^{*}$.

(c) Newton's method applied to the collocation equations converges quadratically in a neighborhood of $u_{\pi}$ for $h \leqslant \delta$. 
(d) The following error estimates hold for $r=0,1$ :

$$
\left|D^{r}\left(u-u_{\pi}\right)\right| \leqslant \kappa h^{\min (p, k)} .
$$

(e) Furthermore, for the collocation solution of the linearized problem $u_{\pi}^{\text {lin }}$ the following estimates hold:

$$
\left|D^{r}\left(u-u_{\pi}^{l i n}\right)\right| \leqslant \kappa h^{\min (p, k)} \quad \text { and } \quad\left|D^{r}\left(u-u_{\pi}\right)\right|=D^{r}\left(u-u_{\pi}^{l i n}\right)+O\left(h^{2 \min (p, k)}\right) .
$$

Proof. See [5, Theorem 2.1, p. 231] and [4].

For ODEs this is usually the departure point in deriving an even higher order of convergence at mesh points, i.e., superconvergence for special sets of collocation points. A crucial basis for all these results is that the Green's matrix $H\left(x, x_{i}\right)$ has essentially the same smoothness as the solution itself when $x \neq x_{i}$. This property does not hold for DDEs; see [4] for a simple example. As a consequence, superconvergence can not be shown in general. However, under severe restriction of the class of problems considered and for a special construction of the partition $\pi$, superconvergence can be shown to hold. In fact, for superconvergence to occur for DDEs of the form (4.5) the mesh needs to be chosen such that mesh points are mapped into mesh points, and collocation points into collocation points, by the delays. This implies that we can only expect to obtain superconvergence when the $s_{\sigma}$ 's are rationally related.

This has been further analyzed by Bellen [10] for the special case of initial value problems. However, even if superconvergence is lost, the improvement of order of convergence over approximation techniques is still substantial, since approximation theory uses systems of ODEs to approximate the solution of DDEs, and hence attempts to approximate solutions which are only usually piecewise smooth through globally smooth functions (see, for example, [7]).

In contrast with the case of differential equations the error is not necessarily localized when approximating these mixed type delay equations. This impacts the error estimation and hence the mesh selection, and a future improvement will be to address this issue. The error estimation and mesh selection employed are adopted from COLMOD and are described in [42]. Error estimation is based upon the maximum moduli of the left-sided and right-sided approximations to the appropriate derivatives on each subinterval. Mesh selection is based upon so-called semiequidistribution in which the error over each subinterval is approximately equidistributed.

\section{REFERENCES}

[1] K. A. Abell, Analysis and Computation of the Dynamics of Spatially Discrete Phase Transition Equations, Ph.D. thesis, University of Sussex, Brighton, UK, 2001.

[2] U. Ascher, J. Christiansen, And R. D. Russell, Collocation software for boundary-value ODEs, ACM Trans. Math. Software, 7 (1981), pp. 209-222.

[3] U. M. Ascher, R. M. M. MattheiJ, And R. D. Russell, Numerical Solution of Boundary Value Problems for Ordinary Differential Equations, Classics Appl. Math. 13, SIAM, Philadelphia, 1995.

[4] G. BADER, Numerische Behandlung von Randwertproblemen für Funktional-Differentialgleichungen, Ph.D. thesis, University of Heidelberg, Heidelberg, Germany, 1984.

[5] G. BADER, Solving boundary value problems for functional differential equations by collocation, in Numerical Boundary Value ODEs, Progr. Sci. Comput. 5, U. M. Ascher and R. D. Russell, eds., Birkhäuser Boston, Boston, 1985, pp. 227-243. 
[6] G. Bader And U. Ascher, A new basis implementation for a mixed order boundary value ODE solver, SIAM J. Sci. Statist. Comput., 8 (1987), pp. 483-500.

[7] H. T. BAnks And F. KAPPEL, Spline approximations for functional differential equations, J. Differential Equations, 34 (1979), pp. 496-522.

[8] P. Bates And A. Chmaj, On a discrete convolution model for phase transitions, Arch. Ration. Mech. Anal., 150 (1999), pp. 281-305.

[9] J. Bell AND C. Cosner, Threshold behavior and propagation for nonlinear differential-difference systems motivated by modeling myelinated axons, Quart. Appl. Math., 42 (1984), pp. 1-14.

[10] A. Bellen, One-step collocation for delay differential equations, J. Comput. Appl. Math., 10 (1984), pp. 275-283.

[11] W.-J. BEYN, The numerical computation of connecting orbits in dynamical systems, IMA J. Numer. Anal., 9 (1990), pp. 379-405.

[12] J. W. Cahn, J. Mallet-Paret, and E. S. Van Vleck, Traveling wave solutions for systems of ODEs on a two-dimensional spatial lattice, SIAM J. Appl. Math., 59 (1998), pp. 455-493.

[13] J. R. CAsh, G. Moore, And R. W. Wright, An automatic continuation strategy for the solution of singularly perturbed nonlinear two-point boundary value problems, J. Comput. Phys., 122 (1995), pp. 266-279.

[14] S. N. Chow, J. Mallet-Paret, And W. Shen, Traveling waves in lattice dynamical systems, J. Differential Equations, 149 (1998), pp. 248-291.

[15] F. DE Hoog AND R. WeIss, An approximation theory for boundary value problems on infinite intervals, Computing, 24 (1980), pp. 227-239.

[16] C. M. Elliott, A. R. Gardiner, I. Kostin, And B. Lu, Mathematical and numerical analysis of a mean-field equation for the Ising model with Glauber dynamics, in Chaotic Numerics, Contemp. Math. 172, P. E. Kloeden and K. J. Palmer, eds., AMS, Providence, RI, 1994, pp. 217-242.

[17] C. E. Elmer, M. Rodrigo, and R. M. Miura, Constructing Examples: A Technique for Finding Equation/Solution Pairs for Mixed-Type Functional Differential Equations, preprint, New Jersey Institute of Technology, Newark, NJ.

[18] C. E. Elmer And E. S. VAn Vleck, Analysis and computation of travelling wave solutions of bistable differential-difference equations, Nonlinearity, 12 (1999), pp. 771-798.

[19] C. E. Elmer AND E. S. VAN VlECK, Traveling wave solutions for bistable differential-difference equations with periodic diffusion, SIAM J. Appl. Math., 61 (2001), pp. 1648-1679.

[20] C. E. Elmer And E. S. VAn Vleck, A variant of Newton's method for mixed type functional differential equations, J. Dynam. Differential Equations, 14 (2002), pp. 493-517.

[21] K. Engelborghs And E. Doedel, Stability of piecewise polynomial collocation for computing periodic solutions of delay differential equations, Numer. Math., 91 (2002), pp. 627-648.

[22] K. Engelborghs, T. Luzyanina, K. J. In 'T Hout, and D. Roose, Collocation methods for the computation of periodic solutions of delay differential equations, SIAM J. Sci. Comput., 22 (2000), pp. 1593-1609.

[23] B. Fiedler And J. Scheurle, Discretization of Homoclinic Orbits, Rapid Forcing and "Invisible" Chaos, AMS, Providence, RI, 1996.

[24] P. FIFE AND J. MCLEOD, The approach of solution of nonlinear diffusion equations to traveling front solutions, Arch. Ration. Mech. Anal., 65 (1977), pp. 333-361.

[25] B. SAndstede, J. HARTERICh, AND A. ScheEl, Exponential dichotomies for linear nonautonomous functional differential equations of mixed type, Indiana Univ. Math. J., 51 (2002), pp. 1081-1109.

[26] J. P. KeEner, Propagation and its failure in coupled systems of discrete excitable cells, SIAM J. Appl. Math., 47 (1987), pp. 556-572.

[27] J. P. KeEneR, The effects of discrete gap junction coupling on propagation in myocardium, J. Theoret. Biol., 148 (1991), pp. 49-82.

[28] M. LEntini AND H. B. KelleR, Boundary value problems on semi-infinite intervals and their numerical solution, SIAM J. Numer. Anal., 17 (1980), pp. 577-604.

[29] J. MALlet-PAREt, The Fredholm alternative for functional differential equations of mixed type, J. Dynam. Differential Equations, 11 (1999), pp. 1-48.

[30] J. Mallet-PARET, The global structure of traveling waves in spatially discrete dynamical systems, J. Dynam. Differential Equations, 11 (1999), pp. 49-128. 
[31] J. Mallet-Paret and S. Verduyn Lunel, Exponential dichotomies and Wiener-Hopf factorizations for mixed-type functional differential equations, J. Differential Equations, to appear.

[32] H. P. McKean, Nagumo's equation, Advances in Math., 4 (1970), pp. 209-223.

[33] K. J. PAlmer, Exponential dichotomies and transversal homoclinic points, J. Differential Equations, 55 (1984), pp. 225-256.

[34] A. Rustichini, Functional differential equations of mixed type: The linear autonomous case, J. Dynam. Differential Equations, 1 (1989), pp. 121-143.

[35] A. Rustichini, Hopf bifurcations for functional differential equations of mixed type, J. Dynam. Differential Equations, 113 (1994), pp. 145-177.

[36] G. Samaey, K. Engelborghs, And D. Roose, Numerical computation of connecting orbits in delay differential equations, Numer. Algorithms, 30 (2002), pp. 335-352.

[37] B. Sandstede, Convergence estimates for the numerical approximation of homoclinic solutions, IMA J. Numer. Anal., 17 (1997), pp. 437-462.

[38] S. SCHECTER, Rate of convergence of numerical approximations to homoclinic bifurcation points, IMA J. Numer. Anal., 15 (1995), pp. 23-60.

[39] L. F. Shampine, R. C. Allen, And S. Pruess, Fundamentals of Numerical Computing, John Wiley, New York, 1997.

[40] T. Strunz And F.-J. Elmer, Driven Frenkel-Kontorova model I. Uniform sliding states and dynamical domains of different particle densities, Phys. Rev. E (3), 58 (1998), pp. 1601-1611.

[41] T. Strunz And F.-J. Elmer, Driven Frenkel-Kontorova model II. Chaotic sliding and nonequilibrium melting and freezing, Phys. Rev. E (3), 58 (1998), pp. 1612-1620.

[42] R. W. Wright, J. R. CASh, AND G. Moore, Mesh selection for stiff two point boundary value problems, Numer. Algorithms, 7 (1994), pp. 205-224.

[43] B. Zinner, Stability of traveling wavefronts for the discrete Nagumo equation, SIAM J. Math. Anal., 22 (1991), pp. 1016-1020.

[44] B. ZinneR, Existence of traveling wavefront solutions for the discrete Nagumo equation, J. Differential Equations, 96 (1992), pp. 1-27. 